\title{
High-fat diet exacerbates SIV pathogenesis and accelerates disease progression
}

\author{
Tianyu He, ${ }^{1,2}$ Cuiling Xu, ${ }^{1,3}$ Noah Krampe ${ }^{1}$, Stephanie M. Dillon, ${ }^{4}$ Paola Sette, ${ }^{1,3}$ Elizabeth Falwell, ${ }^{1,3}$ George S. Haret-Richter, ${ }^{1,2}$ \\ Tiffany Butterfield, ${ }^{5}$ Tammy L. Dunsmore, ${ }^{1}$ William M. McFadden Jr., ${ }^{1}$ Kathryn J. Martin, ${ }^{1}$ Benjamin B. Policicchio, ${ }^{1,6}$ \\ Kevin D. Raehtz, ${ }^{1,3}$ Ellen P. Penn, ${ }^{1}$ Russell P. Tracy, ${ }^{7}$ Ruy M. Ribeiro,,${ }^{8,9}$ Daniel N. Frank, ${ }^{4}$ Cara C. Wilson, ${ }^{4}$ Alan L. Landay, ${ }^{5}$ \\ Cristian Apetrei, ${ }^{1,3,6}$ and Ivona Pandrea ${ }^{1,2,6}$ \\ 'Center for Vaccine Research, ${ }^{2}$ Department of Pathology, and ${ }^{3}$ Division of Infectious Diseases, Department of Medicine, School of Medicine, University of Pittsburgh, Pittsburgh, Pennsylvania, USA. ${ }^{4}$ Division \\ of Infectious Diseases, University of Colorado, Anschutz Medical Campus, Aurora, Colorado, USA. ${ }^{5}$ Department of Microbial Pathogens and Immunity, Rush University, Chicago, Illinois, USA. ${ }^{6}$ Department of \\ Infectious Diseases and Microbiology, Graduate School of Public Health, University of Pittsburgh, Pittsburgh, Pennsylvania, USA. ${ }^{7}$ Department of Pathology and Laboratory Medicine, University of Vermont, \\ Burlington, Vermont, USA. ${ }^{8}$ Los Alamos National Laboratory, Los Alamos, New Mexico, USA. ${ }^{9}$ Faculdade de Medicina da Universidade de Lisboa, Lisbon, Portugal.
}

\begin{abstract}
Consuming a high-fat diet (HFD) is a risk factor for obesity and diabetes; both of these diseases are also associated with systemic inflammation, similar to HIV infection. A HFD induces intestinal dysbiosis and impairs liver function and coagulation, with a potential negative impact on HIV/SIV pathogenesis. We administered a HFD rich in saturated fats and cholesterol to nonpathogenic (African green monkeys) and pathogenic (pigtailed macaques) SIV hosts. The HFD had a negative impact on SIV disease progression in both species. Thus, increased cell-associated SIV DNA and RNA occurred in the HFD-receiving nonhuman primates, indicating a potential reservoir expansion. The HFD induced prominent immune cell infiltration in the adipose tissue, an important SIV reservoir, and heightened systemic immune activation and inflammation, altering the intestinal immune environment and triggering gut damage and microbial translocation. Furthermore, HFD altered lipid metabolism and HDL oxidation and also induced liver steatosis and fibrosis. These metabolic disturbances triggered incipient atherosclerosis and heightened cardiovascular risk in the SIV-infected HFD-receiving nonhuman primates. Our study demonstrates that dietary intake has a discernable impact on the natural history of HIV/SIV infections and suggests that dietary changes can be used as adjuvant approaches for HIV-infected subjects, to reduce inflammation and the risk of nonAIDS comorbidities and possibly other infectious diseases.
\end{abstract}

\section{Introduction}

Persistent immune activation and inflammation are hallmarks of chronic HIV/SIV infection and strong predictors of disease progression, independent of plasma viral loads ( $\mathrm{pVLs}$ ), or peripheral $\mathrm{CD}^{+} \mathrm{T}$ cell counts (1). They also persist in individuals with HIV/ SIV and nonhuman primates (NHPs) on antiretroviral therapy (ART), predicting poor immune restoration, accelerated aging, and development of non-AIDS-associated comorbidities (2-5). The mechanisms of HIV/SIV-associated immune activation and inflammation are the subject of intense research, with key determinants including persistent virus replication, coinfections, dysregulation of $\mathrm{T}$ cell homeostasis, and microbial translocation (4, 6-8). Meanwhile, other unknown factors likely contribute to HIV/SIV-associated chronic immune activation and inflammation. Among these, dietary habits may affect inflammation through multiple pathways (9), altering the pathogenesis of chronic HIV/SIV infection. Little is known, however, with regard to these effects.

Conflict of interest: The authors have declared that no conflict of interest exists. Copyright: (5) 2019, American Society for Clinical Investigation.

Submitted: March 20, 2018; Accepted: September 10, 2019; Published: November 11, 2019. Reference information: J Clin Invest. 2019;129(12):5474-5488.

https://doi.org/10.1172/JCI121208.
Consuming a high-fat diet (HFD) is a leading risk factor for obesity and type 2 diabetes (10), which are both characterized by chronic inflammation (10), marked by elevated TNF- $\alpha$, IL-1, IL-6, and accumulation of proinflammatory macrophages in the adipose tissue (10). A HFD alters the gastrointestinal (GI) mucosa, disrupts gut microbiota, and induces intestinal damage. These conditions all lead to microbial translocation (11-13), further boosting chronic immune activation and inflammation, thus accelerating HIV/SIV disease progression.

In humans, Western diets are associated with an increased risk of nonalcoholic fatty liver disease (NAFLD) (14). The impact of Western diet on liver health is discernible within weeks (15). In the United States, NAFLD became a significant health problem in male adolescents who frequently consume Western diets (15). HFD can also induce NAFLD in mice and rats, along with significant elevation in TNF- $\alpha, \mathrm{NF}-\kappa \mathrm{B}$, and oxidative stress. These liver alterations correlate with thickening of the carotid intima (16), suggesting a possible connection between liver pathology and cardiovascular (CV) disease. In humans, through elevated inflammation via the TLR4/NF- $\mathrm{KB}$ signaling pathway, HFD causes renal, retinal, and brain function impairments (17). As liver, renal, and neurological disorders are also common HIV-associated comorbidities, HFD may increase the risk for or the severity of HIV comorbidities. 


\section{Table 1. Comparison of ND and HFD composition}

\begin{tabular}{|c|c|c|}
\hline & ND & HFD \\
\hline \multicolumn{3}{|l|}{ Composition $^{\mathrm{A}}$} \\
\hline Fat, \% & 5.0 & 15.2 \\
\hline Cholesterol, ppm & 80 & 946 \\
\hline Linoleic acid, \% & 1.46 & 3.02 \\
\hline Linolenic acid, \% & 0.09 & 0.09 \\
\hline Arachidonic acid, \% & $<0.01$ & 0.01 \\
\hline Omega-3 fatty acids, $\%$ & 0.15 & 0.02 \\
\hline Total saturated fatty acids, \% & 1.36 & 6.05 \\
\hline Total monounsaturated fatty acids, \% & 1.72 & 4.85 \\
\hline Protein, \% & 15.6 & 18 \\
\hline Carbohydrates, \% & 60 & 55.6 \\
\hline \multicolumn{3}{|l|}{ Calories provided by } \\
\hline Protein, \% & 17.96 & 16.7 \\
\hline Fat, \% & 12.95 & 31.8 \\
\hline Carbohydrates, \% & 69.09 & 51.5 \\
\hline
\end{tabular}

HFD, high-fat diet; ND, normal diet; ppm, parts per million. ANutrients are expressed as percentage of ration except where otherwise indicated. Moisture content is assumed to be $10.0 \%$ for the purpose of the calculations. Bolded values represent major differences between HFD and normal chow.

Additionally, HFD is a classical risk factor for CV diseases. HFD induces atherosclerosis (18) by enhancing dyslipidemia and macrophage infiltration in the aorta (18). HFD also alters the coagulation profiles and promotes hypercoagulopathy, by increasing plasma levels of endogenous thrombin and procoagulant factors (e.g., FII, FVII, FVIII, and FXII), or decreased fibrinolysis (19). Hypercoagulability is common in HIV/SIV infections and may contribute to residual immune activation and inflammation (20) even in individuals with ART suppression $(2,3)$. By altering the lipid metabolism and coagulation, HFD may thus enhance the incidence of $\mathrm{CV}$ comorbidities, the most frequent complications, and the leading cause of mortality in HIV-infected subjects (21).

As such, HFD may significantly affect the natural history of HIV/SIV, limit ART efficacy, promote HIV/SIV-related comorbidities, and ultimately increase mortality $(2,3)$. Only 1 study previously reported accelerated disease progression in SIV-infected rhesus macaques receiving HFD (22). The underlying mechanisms and the potential impact on SIV-related comorbidities were not investigated in detail, however, and only the model of pathogenic HIV infection was evaluated.

To thoroughly assess the impact of HFD on HIV/SIV natural history and associated comorbidities, we conducted a comprehensive study in which we administered a HFD rich in saturated fats and cholesterol to 2 NHP models of SIVsab infection: African green monkeys (AGMs), in which infection is nonpathogenic (i.e., lacking gut damage, immune activation and inflammation, comorbidities and disease progression) and pigtailed macaques (PTMs), in which infection is pathogenic (i.e., associating massive gut damage, immune activation and inflammation, comorbidities, progression to AIDS and death).

We report that HFD administration had a major impact on viral reservoir seeding and disease progression in both SIV-in- fected NHP species. HFD also significantly increased systemic immune activation and inflammation and altered the gut mucosal environment, profoundly compromising gut integrity and increasing microbial translocation. Furthermore, HFD induced liver steatosis, fibrosis, and alterations of the lipid metabolism. Finally, HFD increased frequency and severity of CV lesions. Altogether, our results provide strong evidence for the critical role of HFD in modulating HIV/SIV morbidity and mortality.

\section{Results}

Study design. We used a diet that provided 32\% calories from fat (Table 1), as opposed to normal chow, which only provides $13 \%$ calories from fat (Table 1). The composition of the HFD we used closely mimicked that of a typical American diet for the high content of saturated fats and cholesterol (23).

HFD was administered to 2 NHP models of nonpathogenic (AGM) and pathogenic (PTM) SIV infection. The goal was not to compare the HFD impact between species, but rather to assess the HFD consequences within the same species, as direct comparisons between species may be flawed by major intrinsic immunologic and metabolic differences. In the same line of reason, we did not perfectly match the timing of HFD administration in the 2 species.

Four AGMs received HFD from 102 days before infection and up to 200 days after infection (dpi) (Figure 1). One HFD-treated AGM died during the follow-up. The remaining 3 AGMs were released at the study completion. Nine historical control AGMs were used (Figure 1). They were SIV infected in similar conditions, had a similar follow-up and sampling schedule to the AGMs receiving HFD, but received a regular chow diet (Table 1). The AGM historical controls were not subjected to intestinal and liver resections.

Five PTMs received HFD from 39 days before SIV infection until they progressed to AIDS (Figure 1). Ten PTMs were used as controls (Figure 1). They were SIV infected in similar conditions but received a regular chow diet (Table 1). Three PTMs were contemporary controls and had a follow-up and sampling schedule similar to that for HFD-receiving PTMs (including intestinal and liver resections). They either progressed to AIDS or were euthanized when the last HFD-receiving PTM died with AIDS. The remaining 7 PTMs were historical controls. They had a follow-up and sampling schedule similar to that for HFD-receiving PTMs, but were not subjected to intestinal and liver resections. They were euthanized when they progressed to AIDS. All controls received the same chow diet.

HFD has a major impact on disease progression and survival of SIV-infected NHPs. First, we questioned whether HFD affected key parameters of HIV/SIV infection (i.e., CD $4^{+} \mathrm{T}$ cell counts and pVLs).

Peripheral CD $4^{+} \mathrm{T}$ cell loss was significantly more prominent in the SIVsab-infected AGMs receiving HFD compared with controls. As such, although a trend toward recovery was observed during chronic infection, the $\mathrm{CD}^{+} \mathrm{T}$ cell counts were still significantly lower in HFD-receiving AGMs compared with controls (Figure 2A). Interestingly, a CD4 ${ }^{+} \mathrm{T}$ cell decrease trend occurred in AGMs after HFD administration even before SIV infection, which was not due to $\mathrm{CD}^{+} \mathrm{T}$ cell downregulation (24), as no increases in the CD8- $\alpha^{\text {low }}$ or $\mathrm{CD} 4^{\text {neg }} \mathrm{CD} 8^{\text {neg }} \mathrm{T}$ cells were observed in the HFD-treated AGMs during the follow-up (data not shown). 


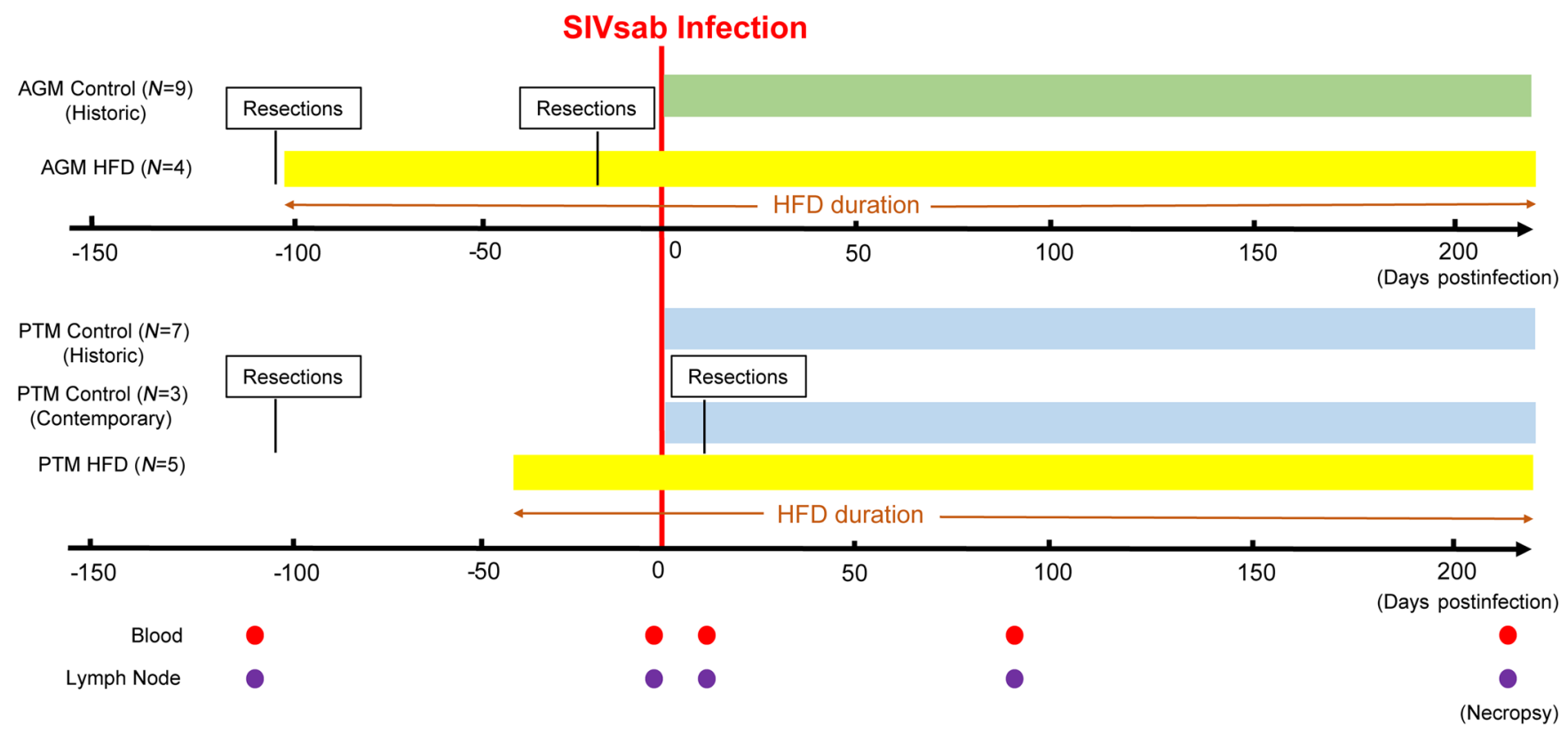

Figure 1. The study design for SIVsab: HFD administration and sampling schedule. A total of 13 AGMs and 15 PTMs were infected with 300 TCID50 of SIVsab. Four AGMs initiated HFD at 102 days before infection and maintained it until 200 days after infection (dpi). Nine historical AGM controls were included and received normal chow. Five PTMs initiated HFD at 39 days before infection and maintained it until necropsy (128-218 days after infection). Three contemporary and 7 historical controls were also included and received normal chow. Sampling of the AGMs and PTMs in the study and control groups was performed as shown.

HFD-receiving AGMs had higher plasma VLs, which reached significance during chronic infection (Figure 2B), along with significantly higher PBMC-associated SIV DNA levels during acute infection (Figure 2C), suggesting that HFD may potentiate early reservoir seeding. This effect was confirmed on the gut samples from the HFD-receiving AGMs, in which acute proviral DNA (vDNA) levels (Figure 2D), along with acute and chronic viral RNA (vRNA) levels (Supplemental Figure 1A; supplemental material available online with this article; https://doi.org/10.1172/JCI121208DS1) were significantly higher than in controls. The acute vRNA levels were also higher in the lymph nodes (LNs) of the HFD-receiving AGMs than in controls (Supplemental Figure 1B).

In SIVsab-infected PTMs, which experience severe $\mathrm{CD} 4^{+} \mathrm{T}$ cell depletion throughout infection, HFD induced further significant $\mathrm{CD}^{+} \mathrm{T}$ cell loss at late time points (Figure 2E). Neither pVLs nor PBMC or intestine cell-associated vDNA levels were significantly increased in the HFD-receiving PTMs compared with controls (Figure 2, F-H). We detected significantly higher cell-associated vRNA levels during chronic infection in both intestine (Supplemental Figure 1C) and LNs (Supplemental Figure 1D) in the HFD-receiving PTMs, however, suggesting higher viral replication in tissues. Note that SIVsab-infected PTMs undergo massive viral replication and yield very high VLs (25), which likely masked the HFD effect on VL.

These data suggest that HFD increases virus replication, particularly in tissues and may thus have an impact on the viral reservoir size.

HFD affected SIV survival in both species (Figure 2, I and J). Although the limited sample size precluded reaching statistical significance, there was a clear trend toward faster progression to AIDS in HFD-receiving PTMs compared with controls $(P=0.0522)$
(Figure 2I). AIDS-defining histological lesions also occurred earlier in HFD-receiving PTMs (Supplemental Table 1). At necropsy, the HFD-receiving PTMs presented with more lymphoid depletion, opportunistic infections, and macrophage infiltration in tissues compared with controls sacrificed at the same time points after infection. The CV lesions were also more severe in the HFD-receiving PTMs (Supplemental Table 2). Altogether, these features indicate an early progression to AIDS and development of more severe SIV-related comorbidities in the HFD-treated animals.

Most strikingly, despite the nonpathogenic nature of SIVsab infection in AGMs, 1 HFD-receiving AGM died during follow-up (Figure 2J), with multiple biomarkers predicting HIV/SIV disease progression increasing in the range found in PTMs with end-stage disease: mucosal (Figure 3A) or systemic (Figure 3B) T cell activation, LPS (Figure 3C), hyaluronic acid (Figure 3D), soluble intercellular adhesion molecule-1 (Figure 3E); and D-dimer (Figure 3F). At necropsy, this AGM presented with lesions representative of AIDS (opportunistic infections) and SIV-related comorbidities: enteritis characterized by a severe disruption of the small intestine architecture with epithelial lesions (Figure 3G) and lamina propria infiltration with foamy cells (Figure 3H). Such sheets of foamy macrophages in the lamina propria are characteristic of atypical mycobacteria infection of the severely immunosuppressed hosts. Although the acid-fast staining could not identify the bacilli in the gut, occurrence of liver granulomas in this animal also support the diagnosis of atypical mycobacteria infection (Figure 3I).

Severe infiltrative cystoisosporiasis was also diagnosed in this AGM (Figure 3J) and confirmed through Giemsa staining (Figure $3 \mathrm{~K})$. The lesions found in the intestinal epithelium and lamina propria are pathognomonic for infection with Cystoisospora belli (for- 
A

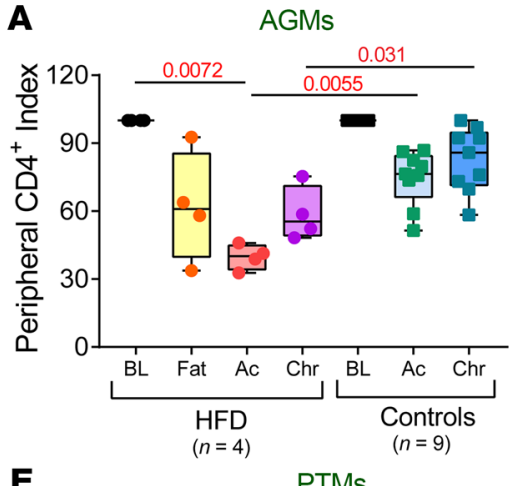

E

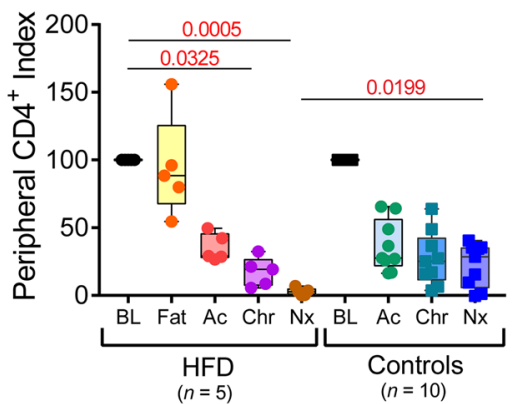

I

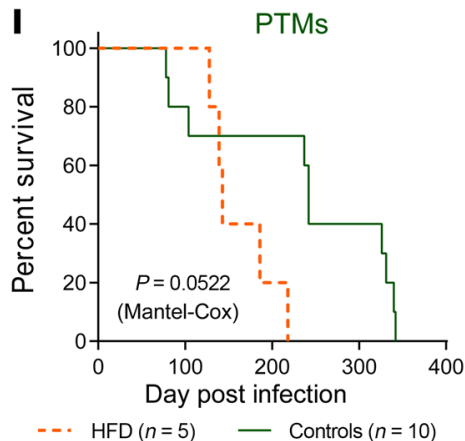

B

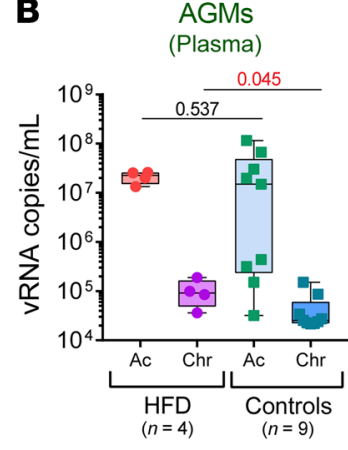

$\mathbf{F}$

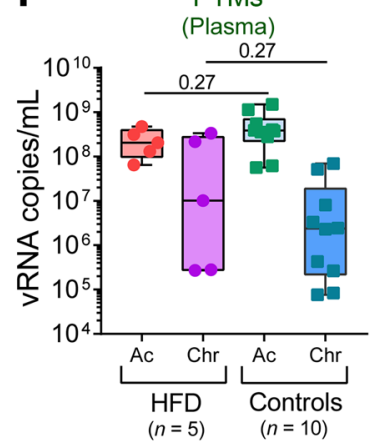

J

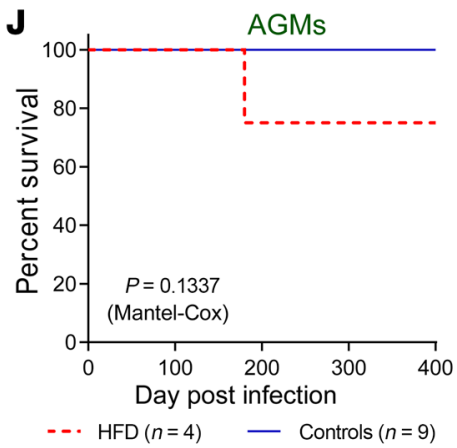

C

G

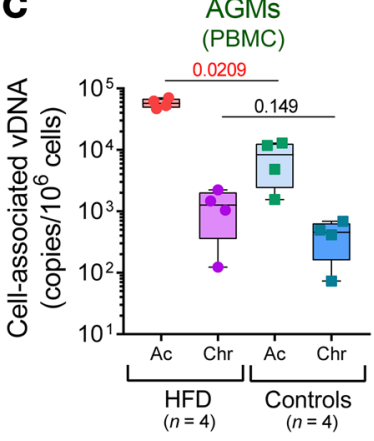

D $\quad$ AGMs

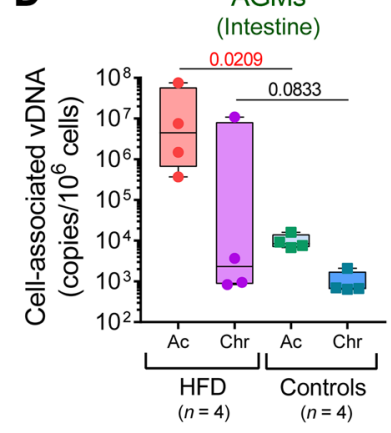

PTMs

(PBMC)

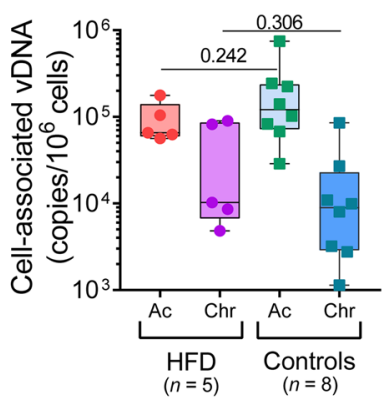

H PTMS

(Intestine)

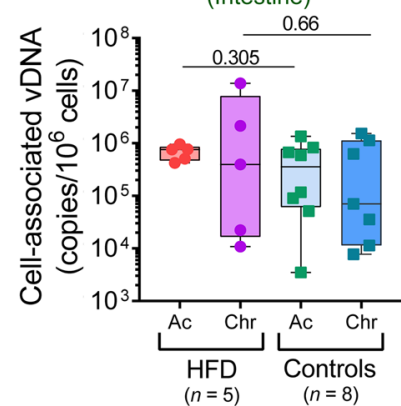

Figure 2. HFD accelerates disease progression and affects survival of SIV-infected NHPs. Peripheral CD4 ${ }^{+}$T cell depletion in SIV-infected AGMs (A) and PTMs (E) are shown as index of baseline levels and compared at key time points of SIV infection within HFD group with the Friedman test corrected for multiple comparisons and between HFD and control groups with Kruskal-Wallis test. Plasma viral loads (B), PBMC-associated viral DNA (C), and intestinal cell-associated DNA (D) levels in SIV-infected ACMs, and in SIV-infected PTMs (F-H) were compared at acute and chronic infection between HFD and control groups with Kruskal-Wallis test. Data are presented as individual values with medians. Sample size $(n)$ and $P$ values are presented on graphs. BL, baseline (preinfection pre-HFD); Ac, acute infection; Chr, chronic infection; Fat, preinfection post-HFD; Nx, necropsy. Kaplan-Meier survival curves of SIV-infected PTMs (I) and AGMs (J) and PTMs are illustrated. Comparison between survival curves of HFD and control groups is performed with Mantel-Cox method. $P$ values are presented on graphs.

merly known as Isospora belli) and were identified, as usually done in a routine histopathological exam, using HE staining (Figure 3J). Diverse evolutionary forms of the parasite were easily recognizable in vacuoles located in the cytoplasm of the gut epithelial cells (Figure 3, $\mathrm{J}$ and $\mathrm{K}$ ). The vacuoles containing the parasites were most frequently located under the host cells' nuclei that were sometimes pushed horizontally creating the classical T-shape form. The parasites were associated with eosinophil infiltration in the lamina propria (Figure 3L). Cystoisosporiasis is an opportunistic infection found in patients infected with HIV who have severe immunosuppression (26).

Furthermore, the AGM progressor also presented with colitis that associated crypt atrophy and infiltration of all intestinal layers with mononuclear cells (Figure 3M). CMV-positive cells were iden- tified in the colon by immunohistochemistry (Figure 3N, upper panels). The CMV infection in the colon was also confirmed through PCR (Supplemental Figure 2). Large, sometimes multinucleated cells with viral cytoplasmic or nuclear inclusions surrounded by a clear halo (Figure 3N, lower panels) also indicative of CMV infection were present in the lung along with interstitial inflammation (Figure 3O). CMV infection of the lung was confirmed by PCR (Supplemental Figure 2). The same technique identified CMV in the spleen and submandibular LNs (Supplemental Figure 2), and the virus could be quantified in blood (at $67 \mathrm{vDNA}$ copies/mL of plasma), thus confirming the disseminated CMV infection.

Finally, chronic glomerulonephritis and thrombotic microangiopathy (TMA), that is, numerous microthrombi in the kidney and other tissues (Figure 3P), was also found in the AGM progressor. 
A

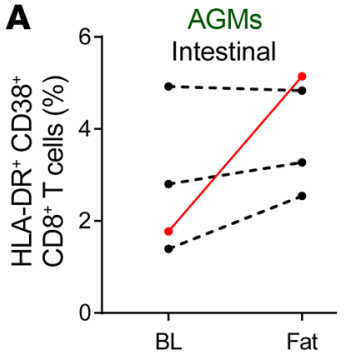

D

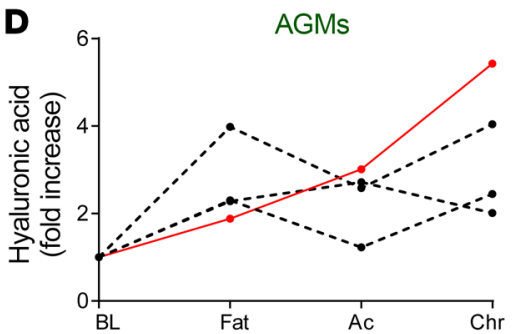

$\mathbf{B}$

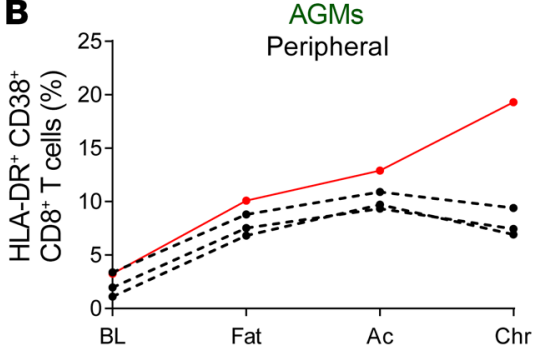

E

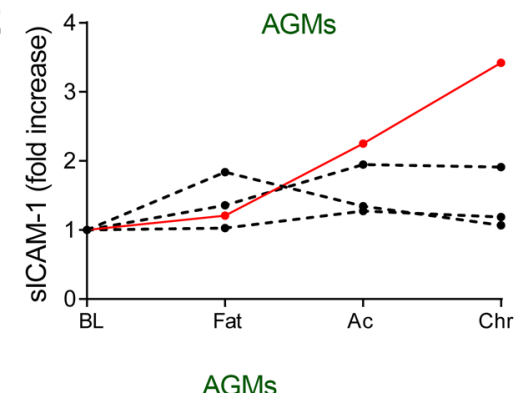

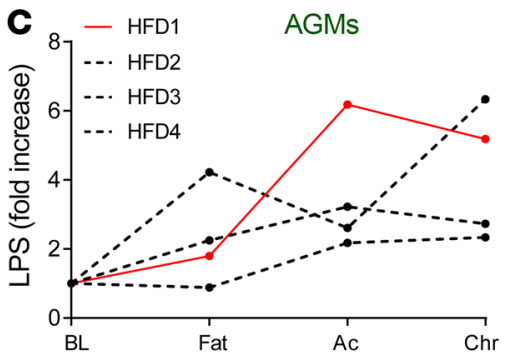

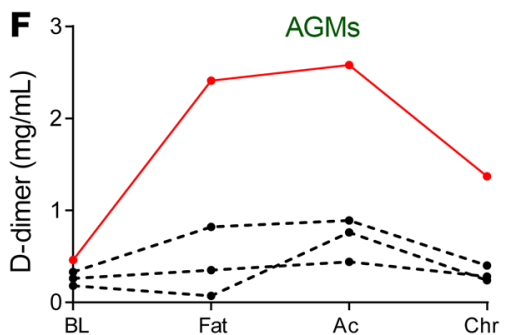

G

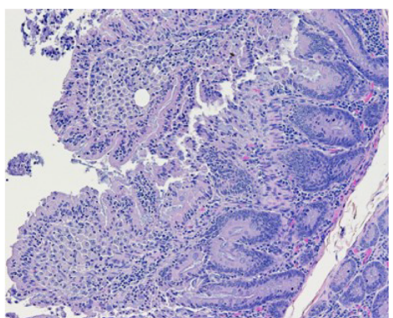

K

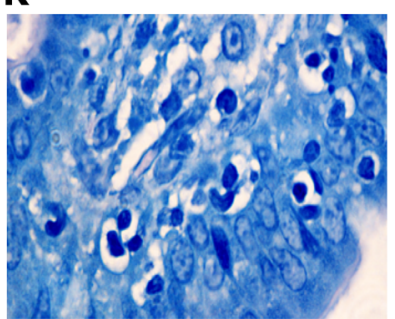

o

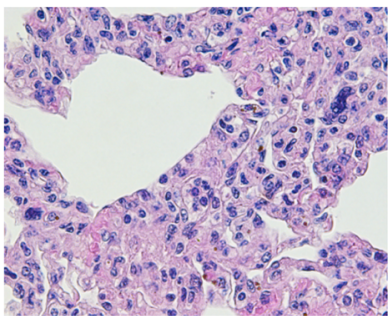

H

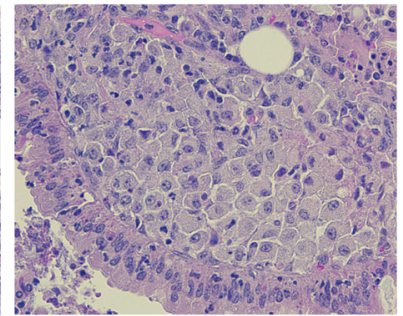

$\mathbf{L}$

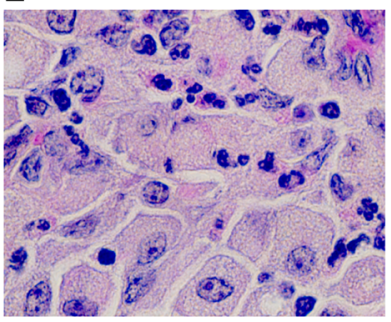

$\mathbf{P}$

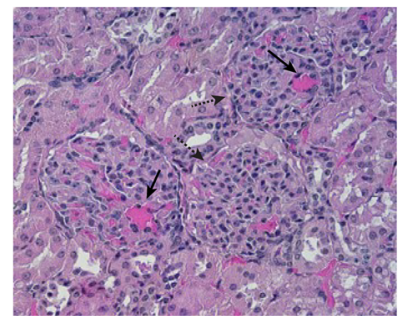

I
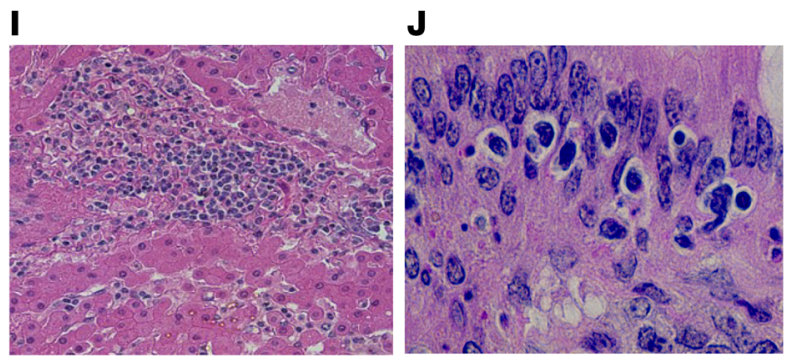

M

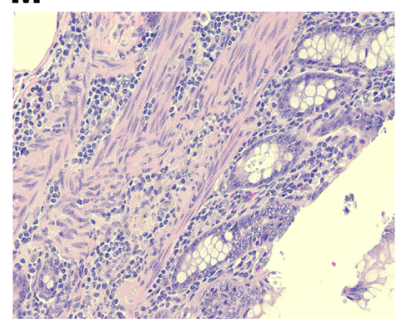

Q

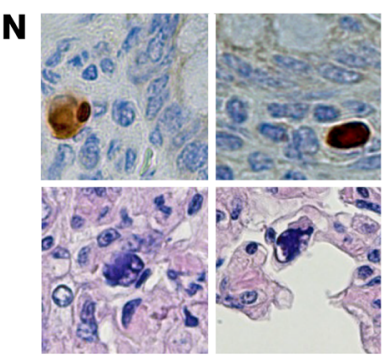

$\mathbf{R}$

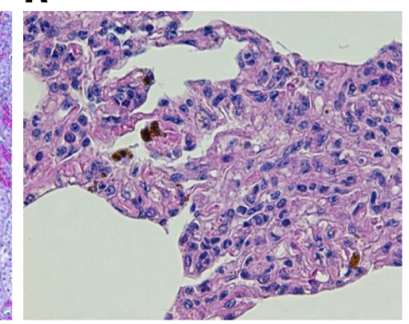

Figure 3. Elevated biomarkers and pathologic changes of the HFD-receiving AGMs that progressed to AIDS-like disease. HFD-receiving ACM HFD1 developed an AIDS-like disease, with distinctly elevated levels of HLA-DR and CD38 coexpressing CD8 ${ }^{+}$T cells in the intestine (A) and periphery (B), LPS (C), hyaluronic acid (D), sICAM-1 (E), and D-dimer (F), as compared with the remaining 3 ACMs on HFD. Ac, acute infection; BL, baseline (preinfection pre-HFD); Chr, chronic infection; Fat, preinfection post-HFD. Representative H\&E images of lesions for AIDS and SIV-associated comorbidities from HFD1. (G) Enteritis, with disrupted intestinal architecture, irregular crypts, enlarged villi, and alteration of the surface epithelium. (H) Numerous foamy macrophages in the lamina propria of the small intestine suggestive for active atypical mycobacterial disease. (I) Hepatic granulomas, suggestive of atypical mycobacterial disease. (J) Cystoisospora belli present in vacuoles in the intestinal epithelium. (K) Giemsa-staining confirmation of small intestine C. belli infection. (L) Eosinophil infiltration in the small intestine, characteristic for parasitic infections. (M) Colitis, with disrupted intestinal architecture, atrophic crypts and mononuclear cell infiltration of the mucosa, submucosa, and of the muscle layers. (N) Upper panels: CMV immunohistochemistry, with positive cells in the colon. Lower panels: Numerous large cells in the lung, with nuclear and cytoplasmic viral inclusions characteristic for CMV infection (detail of $\mathbf{0}$ ). (0) Interstitial pneumonia, with thickened alveolar walls due to infiltration with mononuclear cells. (P) Numerous microthrombi in the kidney glomeruli indicative of TMA (solid arrow). Disrupted kidney cortex architecture with irregular, small fibrotic glomeruli, pathognomonic for chronic glomerulonephritis (dashed arrow). (Q) Enlarged capillaries in the kidney parenchyma indicative of chronic stasis associated with heart failure. (R) Numerous hemosiderin-laden macrophages in the lung, indicative of chronic stasis associated with heart failure. Original magnifications: $\times 200(\mathbf{G}-\mathbf{I}, \mathbf{M}$, and $\mathbf{O}-\mathbf{R}) ; \times 400(\mathbf{L}) ; \times 600(\mathbf{J}, \mathbf{K}$, and $\mathbf{N})$. 
A

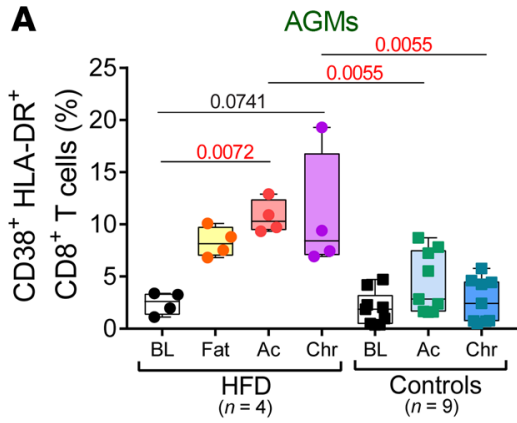

D

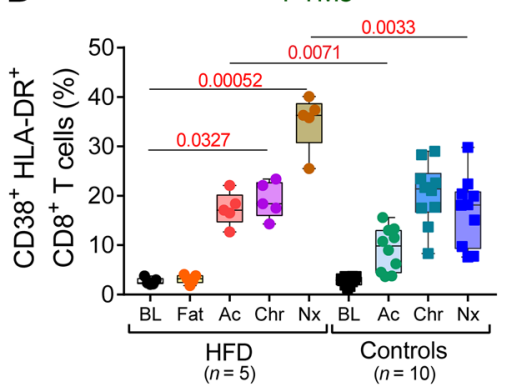

B

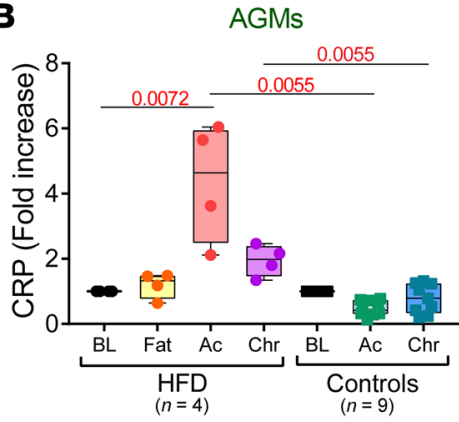

E

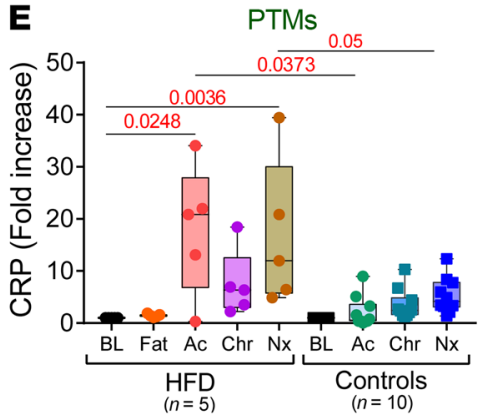

C

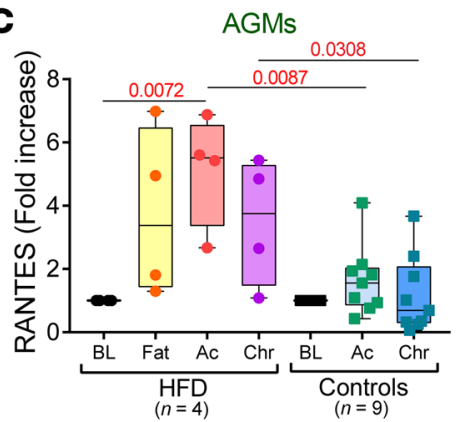

$\mathbf{F}$

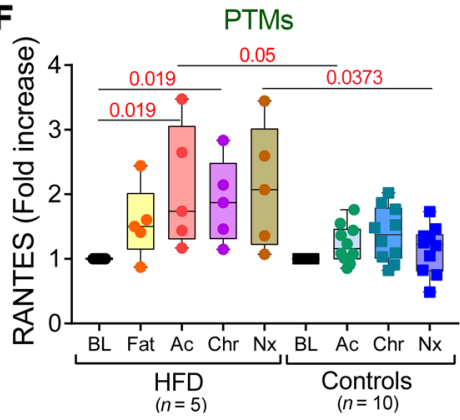

Figure 4. HFD induces elevated systemic immune activation and inflammation in SIV-infected NHPs. Frequencies of CD38 and HLA-DR coexpressing $\mathrm{CD8}^{+}$T cells (A), CRP levels (B) and RANTES levels (C) to baseline levels in AGMs, as well as in PTMs (D-F), are compared at key time points of SIV infection within HFD group with Friedman test corrected for multiple comparisons, and between HFD and control groups with the Kruskal-Wallis test. Data are pre-

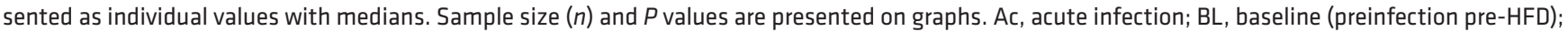
Chr, chronic infection; Fat, preinfection post-HFD; Nx, necropsy.

TMA occurs in progressive HIV/SIV infections, where it is associated with increased levels of D-dimer (3). The AGM progressor had D-dimer levels in the range of PTMs with end-stage disease (Figure $3 \mathrm{~F}$ ). Chronic stasis (characteristic for congestive heart failure) was found in the liver, kidneys (Figure 3Q), and lungs (Figure 3R) and was characterized by dilated capillaries, increased blood content, and numerous hemosiderin-laden macrophages (Figure 3R).

HFD exacerbates systemic immune activation and inflammation. Immune activation and inflammation are hallmarks of chronic pathogenic HIV/SIV infections (1). As HFD also increases inflammation independently (10), we next asked whether HFD and SIV infection can synergistically drive immune activation and inflammation. While SIVsab-infected AGMs typically do not exhibit systemic immune activation and inflammation (1), the frequency of $\mathrm{CD}^{+}$(Figure 4A) and $\mathrm{CD}^{+}$(Supplemental Figure 3A) T cells expressing $\mathrm{HLA}_{-} \mathrm{DR}^{+}$and $\mathrm{CD}_{3} 8^{+}$increased immediately in AGMs after starting the HFD, remaining significantly elevated throughout the follow-up period, with the highest levels of CD38 ${ }^{+} \mathrm{HLA}-$ $\mathrm{DR}^{+} \mathrm{T}$ cells being observed in the AGM progressor (Figure 3, A and $\mathrm{B}$ ). The $\mathrm{Ki}-67^{+} \mathrm{CD} 4^{+} \mathrm{T}$ cells were also significantly increased during both acute and chronic infection (Supplemental Figure 3B) in HFD-receiving AGMs compared with controls.

SIV-infected PTMs receiving HFD similarly experienced a significant increase of the HLA-DR ${ }^{+} \mathrm{CD} 38^{+} \mathrm{CD} 8^{+} \mathrm{T}$ cells during both acute and chronic infection (Figure 4D).

The levels of the systemic inflammation biomarker $\mathrm{C}$ reactive protein (CRP) were significantly higher in HFD-receiving SIV-infected AGMs and PTMs compared with controls (Figure 4, $B$ and E). The levels of RANTES, a biomarker of macrophages and $\mathrm{T}$ cell recruitment at atherosclerotic lesion sites (27), were also significantly elevated compared with controls in both species (Figure 4, C and F).

HFD induces inflammation and immune infiltration in the adipose tissue. Adipose tissue is an important HIV reservoir (28). Prompted by our results showing both higher levels of cellassociated vRNA as well as vDNA and increased acute and chronic immune activation and inflammation in the HFD-receiving NHPs, we further investigated the inflammatory status of the adipose tissue. In SIV-infected PTMs, HFD was associated with more severe inflammatory infiltrates in the adipose tissue surrounding critical organs (GI tract and the heart), compared with SIV-infected controls. High-degree inflammatory infiltrates, represented by mononuclear cells, were observed in the peritoneal fat (Figure 5, left panel) and the epicardial adipose tissue (Figure 5, right panel). Severe inflammatory infiltrates in the epicardium penetrated in the tunica adventitia of the adjacent coronary arteries. In 1 HFD-treated PTM, inflammatory infiltrates penetrated the entire coronary wall, inducing significant damage and promoting leukocyte adhesion (Figure 5, right panel, arrow).

Increased metabolic activity may be a source of the immune activation observed in HFD-receiving NHPs. Increased cell metabolism may drive immune activation. Using the seahorse technology, we assessed the impact of HFD on the metabolic status in PTMs and showed that HFD altered the PBMC metabolic state even before SIV infection. We quantified aerobic glycolysis by monitoring the extracellular acidification rate (ECAR) at basal respiration and in response to oligomycin (to measure ATP production), fluorocarbonyl cyanide phenylhydrazone (to measure maximal respiration), 

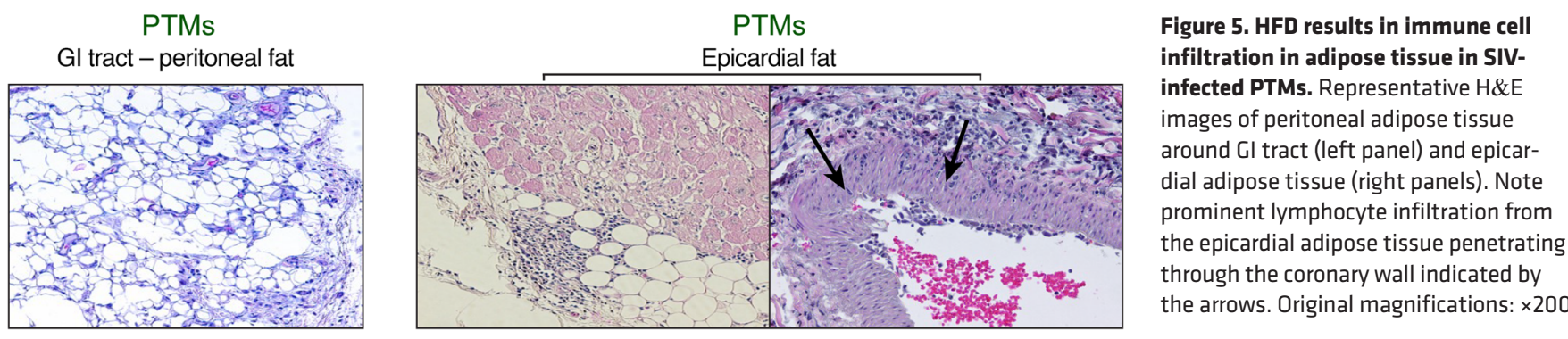

and rotenone addition (to measure nonmitochondrial respiration) (Supplemental Figure 4A). PBMCs collected after administering the HFD had significantly higher AUC of ECAR compared with PBMCs collected before HFD (Supplemental Figure 4B). The oxygen consumption rate (OCR) was also measured to quantify mitochondrial oxidative phosphorylation. After HFD, PBMCs had a trend of a lower OCR/ECAR ratio at basal respiration (Supplemental Figure 4C), indicating a metabolic shift toward aerobic glycolysis. After HFD, PBMCs also showed moderately increased maximal respiration OCR, spare respiratory capacity and ATP production, albeit without reaching significance $(P>0.1)$ (Supplemental Figure 4D). These results collectively indicate a post-HFD PBMC metabolic shift toward aerobic glycolysis, reflecting and contributing to a heightened activation status.

HFD compromises gut immune integrity, promoting microbial translocation. A major contributor to HIV-/SIV-related immune activation and inflammation is gut dysfunction and the consequent microbial translocation (6). Because the HFD-induced changes could potentially disrupt the intestinal immune homeostasis and epithelial barrier integrity, we next assessed HFD impact on the intestinal immune environment and microbial translocation. In HFD-receiving AGMs, intestinal $\mathrm{CD}^{+}{ }^{+} \mathrm{T}$ cells were lost even before infection, yet without reaching statistical significance $(P=0.4029)$, probably due to limited sample size (Figure 6A). Similar to blood, this decrease was not due to $\mathrm{CD} 4^{+} \mathrm{T}$ cell downregulation (24), as no increase of the mucosal CD8- $\alpha^{\text {low }} /$ $\mathrm{CD} 4^{\text {neg }} \mathrm{CD} 8^{\text {neg }} \mathrm{T}$ cells occurred in the HFD-treated AGMs (data not shown). $\mathrm{CD}^{+} \mathrm{T}$ cell loss involved all memory subsets (Figure $6 \mathrm{~B}$ ). Intestinal $\mathrm{CD}^{+} \mathrm{T}$ cell recovery was minimal in chronically SIVinfected HFD-receiving AGMs (to $22.5 \% \pm 4.5 \%$ of baseline levels), while being moderate in controls (to $47.5 \% \pm 5.9 \%$ of baseline levels) (Figure 6A), as reported (8). In PTMs, the magnitude of the SIVsab-induced mucosal $\mathrm{CD} 4^{+} \mathrm{T}$ cell depletion obscured any difference between HFD-treated PTMs and controls.

Among the mucosal $\mathrm{CD}^{+} \mathrm{T}$ cell subsets, Tregs showed a decreasing trend in HFD-fed AGMs even before infection ( $P=$ 0.125) (Supplemental Figure 5A). In PTMs, mucosal CD $4^{+}$Tregs were significantly depleted throughout SIV infection (Supplemental Figure 5B), being significantly lower in the HFD-treated PTMs compared with controls during both acute and chronic infection. Treg decreases could diminish mucosal tolerance to intestinal pathogens and disrupt local inflammation in the gut in the HFD-receiving NHPs (29).

HFD also induced increased activation of mucosal immune cells in HFD-receiving PTMs, with increases of Glut- $1^{+} \mathrm{CD} 38^{+}$ HLA-DR ${ }^{+} \mathrm{CD}^{+} \mathrm{T}$ cells (Figure $6 \mathrm{C}$ ) and macrophage activation, indicated by the increased expression of CD80 (Figure 6D) and CD86 (Figure 6E), especially during chronic SIV infection.

Furthermore, HFD also significantly increased myeloperoxidase $^{+}$neutrophil infiltrates associated with more pronounced epithelial lesions and a higher frequency of crypt abscesses in chronically SIV-infected PTMs (Figure 7A), suggesting greater intestinal barrier damage and stronger inflammatory responses due to heightened microbial translocation.

Systemic biomarkers of microbial translocation and the associated mucosal damage increased post-HFD administration. Plasma LPS levels increased in HFD-receiving AGMs during both acute and chronic SIV infection (Figure 7B) and were highest in the AGM progressor (Figure 3C). No differences in LPS levels were observed between the HFD-receiving and control PTMs, likely due to the massive SIV-induced intestinal damage, or to intrinsically elevated LPS levels in PTMs (30).

Additionally, intestinal fatty acid-binding protein (I-FABP), which is released by necrotic enterocytes, increased even prior SIV infection and remained increased throughout the follow-up in the HFD-receiving AGMs (Figure 7C). I-FABP was also significantly elevated in the HFD-receiving PTMs, especially during acute SIV infection and at the time of progression to AIDS (Figure 7D). These results demonstrate that HFD exacerbated intestinal dysfunction in both NHP models.

HFD induces pathological alterations of the liver. Previous studies showed that HFD affects liver integrity. In particular, HFD-induced low systemic endotoxemia is responsible for NAFLD (31). In our study, steatohepatitis occurred in AGMs 82 days after HFD administration, prior to SIV infection (Figure 8A). Liver steatosis also occurred in all the HFD-receiving PTMs (Figure 8A) only 49 days after HFD (at 9 dpi). Because these PTMs were at a very early stage of SIV infection, NAFLD was due to diet rather than SIV infection. Conversely, NAFLD occurred in only a small fraction of controls and only during a late stage of SIV infection. The fat droplet infiltrates were diffusely distributed throughout the hepatic lobules of the HFD-receiving PTMs (Figure 8A), whereas in controls, they most frequently occurred at the periphery of the hepatic lobule, pathognomonic for hypoxia. The different distribution of fat accumulation in the liver points to different mechanisms for liver steatosis in chronically SIV-infected HFD-receiving PTMs and controls. NAFLD associated moderate sinusoidal fibrosis that became significant during chronic infection, as histologically shown by collagen quantification (Figure 8B), and confirmed by measuring plasma hyaluronic acid (32), which significantly increased in HFD-receiving AGMs and PTMs compared with controls (Figure 8, C and D). Notably, the AGM progressor had 

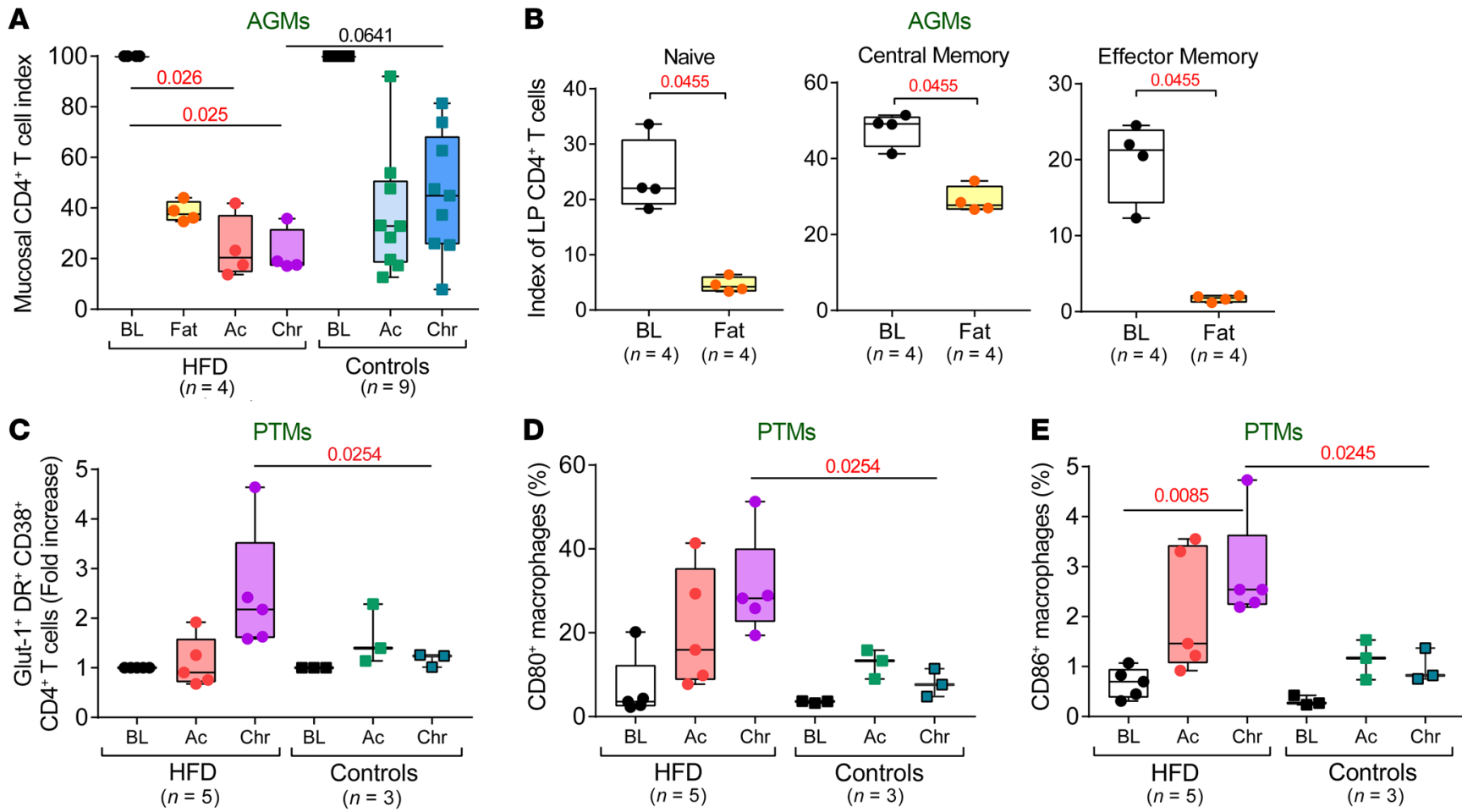

Figure 6. HFD alters gut immune environment and activation states of the immune cells. (A) Mucosal CD4 ${ }^{+} T$ cell depletion in HFD-receiving and control AGMs is shown as an index of baseline levels and compared at key time points of SIV infection within the HFD group with Friedman test corrected for multiple comparisons, and between HFD and control groups with Kruskal-Wallis test. (B) Mucosal-naïve, central memory, and effector memory CD4+ T cells are shown as an index of total baseline mucosal CD4 ${ }^{+} \mathrm{T}$ cell levels and compared before and after HFD in preinfection AGMs with Friedman test. Frequencies of Glut-1, HLA-DR, and CD38 coexpressing CD4+ $T$ cells (C), as well as CD80-expressing (D) and CD86-expressing (E) macrophages in the intestine of PTMs are compared at key time points of SIV infection within HFD group with Friedman test corrected for multiple comparisons and between HFD and control groups with Kruskal-Wallis test. Data are presented as individual values with medians. Sample size $(n)$ and $P$ values are presented on graphs. Ac, acute infection; BL, baseline (preinfection pre-HFD); Chr, chronic infection; Fat, preinfection post-HFD.

the highest levels of plasma hyaluronic acid ( 5.5 -fold increase from baseline) (Figure 3D), pointing to a significant role of HFDinduced liver pathology on the chronic SIV infection outcome.

HFD increases the risk of CV comorbidities in SIV-infected NHPs. $\mathrm{HFD}$, especially with high saturated fat, is a major risk factor for $\mathrm{CV}$ disease (33). We investigated the effects of HFD on the coagulation status and CV health of SIV-infected NHPs, to assess the relative contribution of dietary habits of the HIV-associated CV comorbidities.

The cholesterol (Figure 9A) and oxidized HDL (Figure 9B) levels increased in HFD-receiving, SIV-infected AGMs, while remaining virtually unchanged in controls. Oxidized HDL directly correlates with accelerated atherosclerosis (34); therefore, its post-HFD increase in SIV-infected AGMs (which do not develop $\mathrm{CV}$ disease) indicates harmful effects of HFD on CV health. Soluble tissue factor (sTF) also increased in HFD-receiving, chronically SIV-infected AGMs (Figure 9C), pointing to activation of the extrinsic coagulation pathway and a higher thrombotic risk. The increased $\mathrm{CV}$ risk was also supported by significantly elevated levels of soluble p-selectin (Figure 9D) and soluble intercellular adhesion molecule-1 (sICAM-1) (Figure 9E) throughout SIV infection in HFD-receiving AGMs compared with controls, demonstrating increased platelet and endothelial activation, along with increased immune cell recruitment to the blood vessel walls. The AGM progressor exhibited the highest sICAM-1 (Figure 3E), and D-dimer levels (Figure 3F).
In the pathogenic SIV infection of PTMs, which already associates hypercoagulability (3), HFD administration did not further increase the oxidized HDL or STF, but induced significantly higher FVII levels during SIV infection (Figure 9F). Furthermore, troponin-I was significantly elevated in 3 out of 5 chronically SIV-infected PTMs on HFD, while remaining undetectable in controls (Figure $9 G)$, directly confirming that HFD induced severe cardiac injury.

HFD increases the frequency and severity of CV lesions in SIVinfected PTMs. To assess the frequency of CV tissue lesions in HFD-treated NHPs, we conducted a histopathological diagnostic on the necropsy samples collected from the PTMs. Although the CV lesions found in the HFD-treated PTMs were similar to those in chronic SIV-infected controls, they were more severe and occurred earlier (Supplemental Table 2). CV lesions included mononuclear cell infiltration in myocardium (Figure 10A) and epicardium (Figure 10B), which formed lymphoid aggregates (Figure 10C), severe myocardial cytolysis (Figure 10D), myocardial fatty infiltration (Figure 10E), and myocardial microthrombi (Figure 10F). Two HFD-receiving PTMs had incipient aortic dissection (blood penetration underneath the endothelium) (Figure 10G). These lesions usually evolve into aorta aneurysms that may rupture and induce fatal hemorrhages. Diffuse or localized myocardial fibrosis also occurred (Figure 10H), as well as severe pericardial fibrosis, indicative of chronic pericarditis complicated with colla- 
A

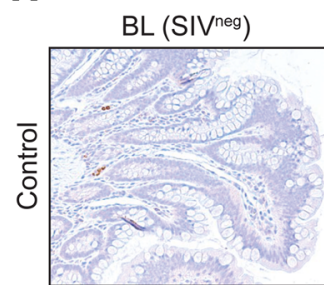

BL (SIV neg pre-HFD)

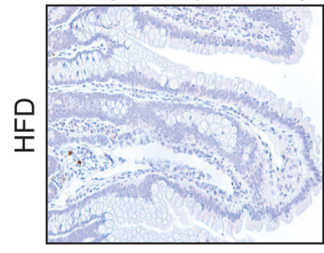

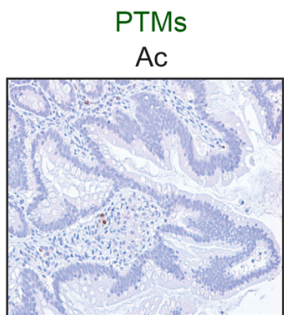

$\mathrm{Ac}$

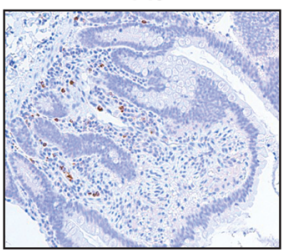

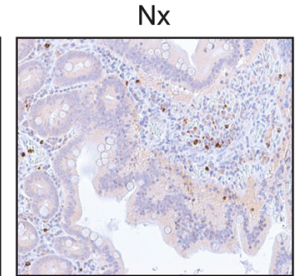

$\mathrm{Nx}$

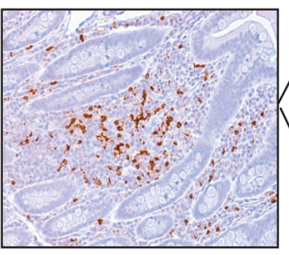

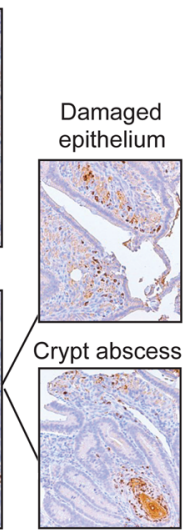

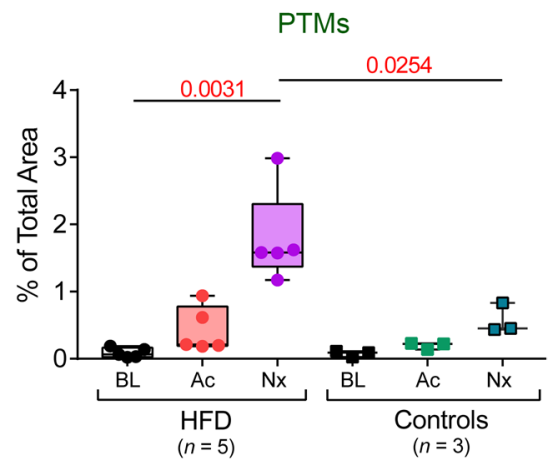

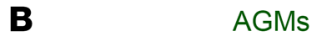

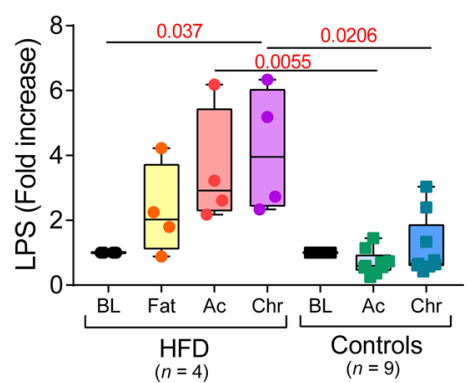

AGMs

C

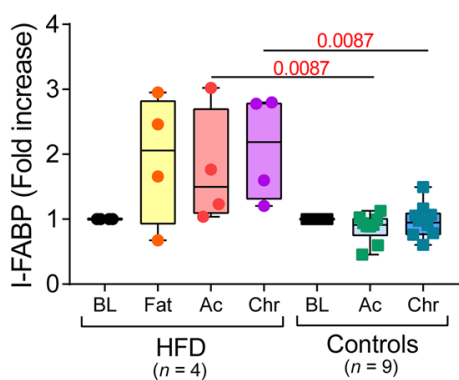

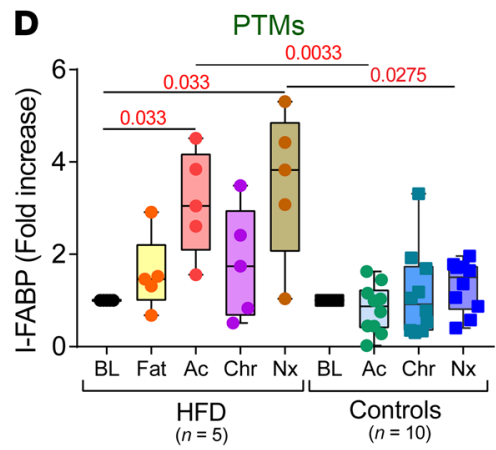

D

Figure 7. HFD induces intestinal epithelial barrier damage and increases microbial translocation. (A) Representative images of jejunum stained for myeloperoxidase (brown) collected before infection, at 9 days after infection, and at necropsy from contemporary controls and HFD-receiving PTMs. Note substantially increased myeloperoxidase underneath the damaged epithelium and adjacent to the crypt abscess. Original magnifications: $\times 200$. Quantification of the percent area of the jejunum positive for myeloperoxidase is shown in the right panel. Fold increase of LPS (B) and intestinal fatty-acid binding protein (C) to baseline levels in AGMs, as well as fold increase of intestinal fatty-acid binding protein (D) to baseline levels in PTMs are compared at key time points of SIV infection within HFD group with Friedman test corrected for multiple comparisons, and between HFD and control groups with Kruskal-Wallis test. Data are presented as individual values with medians. Sample size $(n)$ and $P$ values are presented on graphs. Ac, acute infection; BL, baseline (preinfection pre-HFD); Chr, chronic infection; Fat, preinfection post-HFD; Nx, necropsy.

gen deposition (Figure 10I), which may restrict cardiac function. Myocardial trichrome quantification showed significant increased fibrosis of the cardiac muscle in the HFD-treated PTMs compared with controls (Figure 10, J and K). Incipient atherosclerosis lesions (fatty streaks) in coronaries, abdominal aorta, and carotid arteries were more frequent in the HFD-treated PTMs compared with controls (Figure 10L). Fatty streaks were composed mainly by foamy cells (macrophages that engulfed cholesterol and triglycerides) and mononuclear cells, with $\mathrm{T}$ cells likely accumulating underneath the vascular endothelium (Figure 10L, left), resulting in a thickened blood vessel intima (Figure 10L, middle). Organized microthrombi that trigger additional leukocyte and platelet adhesion also occurred in the HFD-treated animals (Figure 10L, right).

\section{Discussion}

Although the life span of HIV-infected subjects dramatically improved with ART, a higher mortality rate than in uninfected individuals still persists (2). Therefore, the study of non-AIDS comorbidities in long-term ART-suppressed subjects has gained traction (2). As promoters of non-AIDS comorbidities and the accelerated aging (5), the underlying mechanism(s) of HIV-/SIV-associated immune activation and inflammation are being extensively investigated. Thus, intestinal dysfunction was shown to play a central role in driving disease progression (and to be potentially driven) in addition to infection-related causes, by environmental factors, such as lifestyle and dietary intake. Little is known, however, about the synergy between HIV/SIV infection and environmental factors in driving pathogenesis.

Diet effects on HIV infection are difficult to study in humans, due to confounding factors (ART-related dyslipidemia and toxicity, alcohol, tobacco and recreational drug use, sedentary lifestyle), and ethical limitations (experimental alterations of diet may have unknown effects, posing risks to human subjects; invasive sampling needed to assess diet effects on tissues is impossible in humans). NHPs may be employed to circumvent these ethical issues, while enabling the assessment of the impact of different dietary components on SIV pathogenesis through administration of purified diets with a specific composition, staged introduction of various dietary elements, and testing in a strictly controlled environment.

Mansfield et al. previously reported that an atherogenic diet led to accelerated disease progression in rhesus macaques and highlighted the role of IL-18 in enhancing the disease onset (22). 
A

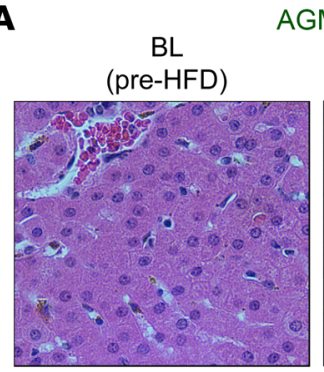

B

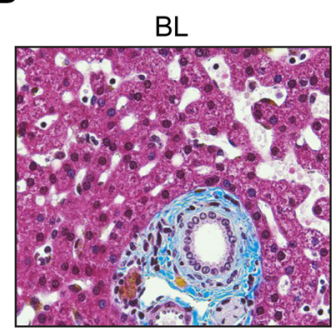

AGMs (82 days post-HFD)

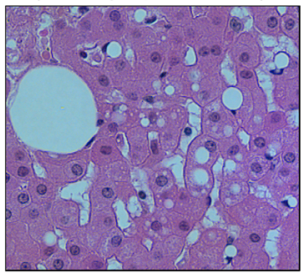

PTMs

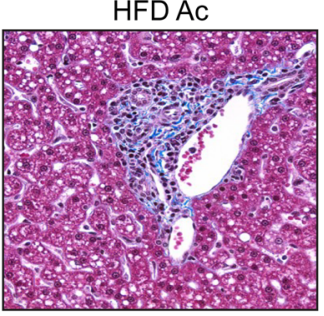

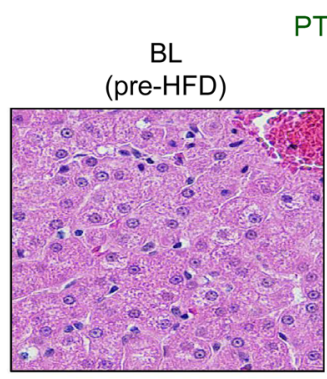

PTMs

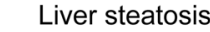

(48 days post-HFD)

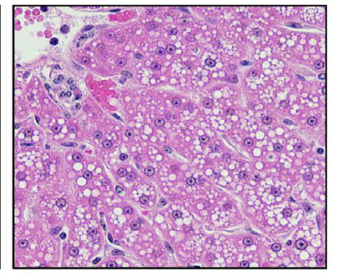

PTMs
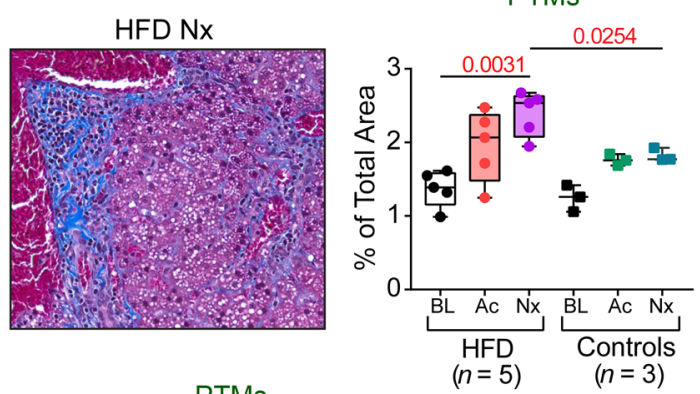

C

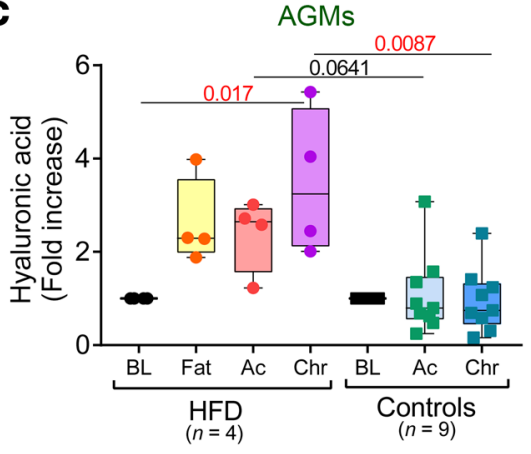

D

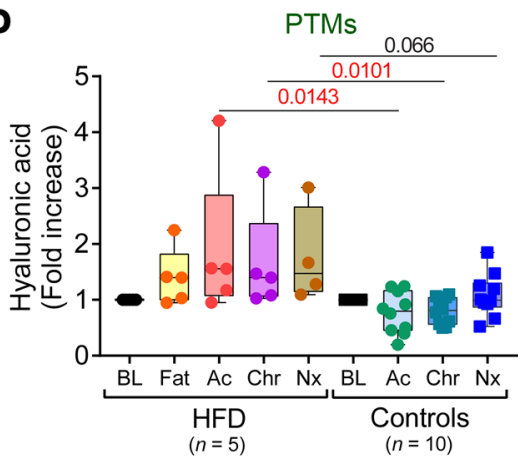

Figure 8. HFD-induced liver steatosis and fibrosis in SIV-infected NHPs. Representative images of HFD-induced liver steatosis in AGMs and PTMs (A). Representative images of liver stained with Masson trichrome showing collagen (blue) collected before infection, at 9 days after infection, and at necropsy from control and HFD-receiving PTMs (B). Original magnifications: $\times 200$. Quantification of the percent area of collagen is shown in the right panel. Fold increase of hyaluronic acid levels vs baseline levels in AGMs (C) and in PTMs (D) are compared at key time points of SIV infection within HFD group with Friedman test corrected for multiple comparisons and between HFD and control groups with Kruskal-Wallis test. Data are presented as individual values with medians. Sample size $(n)$ and $P$ values are presented on graphs. Ac, acute infection; BL, baseline (preinfection pre-HFD); Chr, chronic infection; Fat, preinfection post-HFD; Nx, necropsy.

We reasoned, however, that dietary effects on HIV/SIV pathogenesis and comorbidities are more profound and systemic, so we conducted a comprehensive assessment of the HFD effects on important organ systems, such as gut, liver, and the CV system.

We report that HFD administration to pathogenic (PTMs) and nonpathogenic (AGMs) models of SIVsab infection dramatically affects SIV disease progression and survival. As for RMs (22), the HFD accelerated and exacerbated disease progression in PTMs. Surprisingly, HFD also induced progression to AIDS in an AGM. SIV disease progression is exceptional in natural hosts (35). For this reason, such a dramatic switch toward virulence supports a significant impact of dietary habits on SIV infection outcome, strongly suggesting that diet may be used as an adjuvant to the treatment of HIV/SIV infection and, by extension, to chronic infectious diseases.

Notably, HFD increased viral replication in AGMs and PTMs, as illustrated by plasma VLs and cell-associated SIV RNA from PBMCs, LNs, and intestine. Increased levels of PBMC-associated SIV DNA were also observed during acute SIV infection, when the viral reservoir was being seeded (36). This increased viral replication and reservoir seeding observed in HFD-receiving NHPs may be driven by immune activation and inflammation and may point to a direct HFD impact on HIV/SIV replication and persistence. In turn, increased reservoir seeding during acute SIV infection may further boost systemic immune activation and inflammation, creating a vicious cycle of accelerated disease progression in HFD-treated NHPs.

Adipose tissue is an important, yet neglected, reservoir for HIV/SIV (28). HFD administration induced prominent immune cell infiltration of the adipose tissue around the GI tract (peritoneum) and heart (epicardium and pericardium), creating a proinflammatory environment favoring viral persistence and visceral lesions. Through this mechanism, along with the cholesterol increases, HFD can increase viral reservoir size. As such, HFD may pose a particular threat to long-term suppressors and controllers by increasing the reservoir size, and thus dietary habits should be factored into HIV cure-related research. 

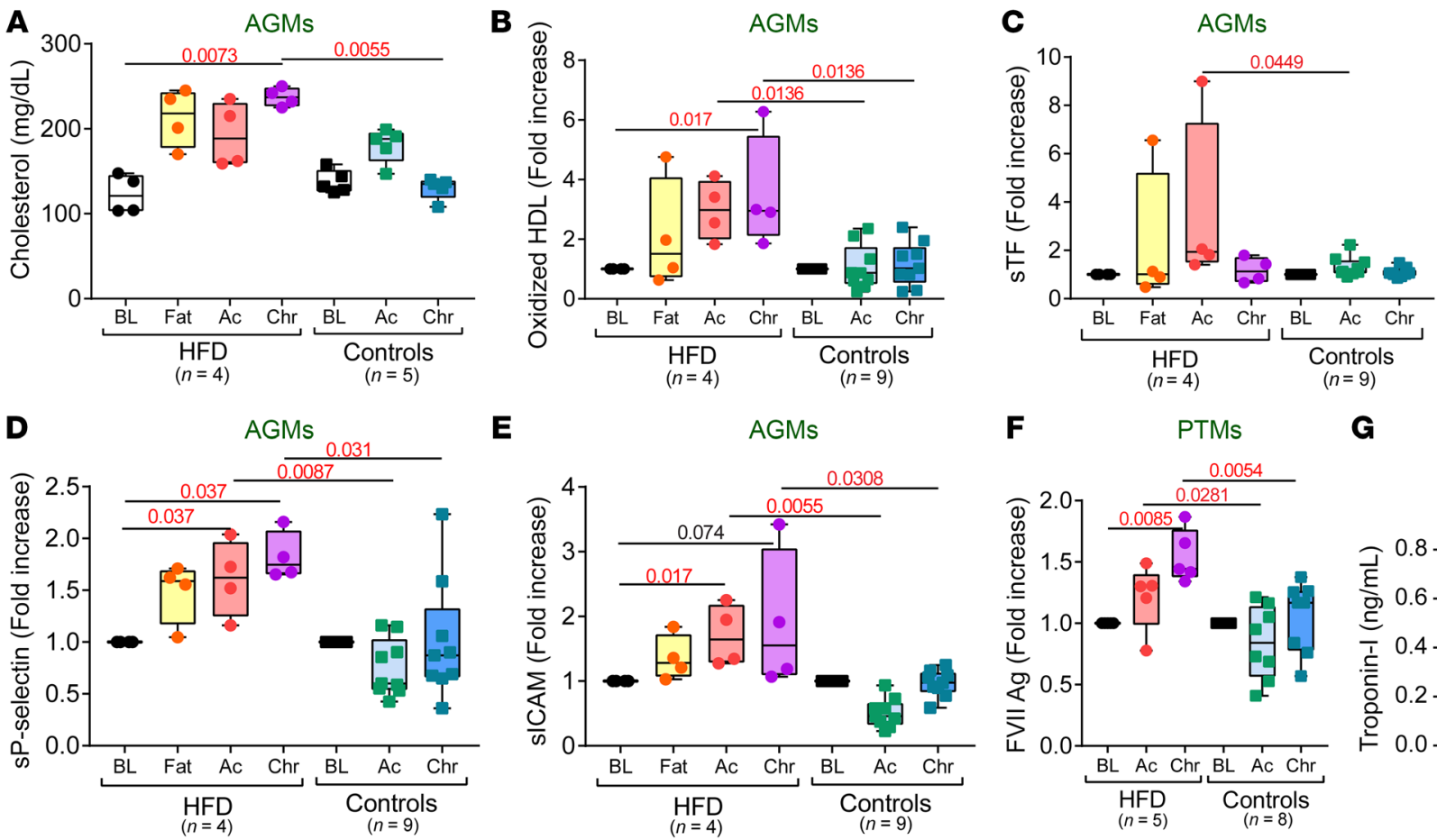

G

PTMs

Figure 9. HFD increases thrombotic cardiovascular risk in SIV-infected NHPs. Serum cholesterol values (A), fold increase of plasma oxidized HDL (B), soluble tissue factor (C), soluble p-selectin (D), and soluble ICAM-1 levels (E) to baseline levels in AGMs, as well as fold increase of factor VII levels to baseline levels in PTMs (F) are compared at key time points of SIV infection within HFD group with Friedman test corrected for multiple comparisons, and between HFD and control groups with Kruskal-Wallis test. (G) Plasma troponin-I levels during chronic infection in PTM are compared between HFD and control groups with Kruskal-Wallis test. Data are presented as individual values with medians. Sample size $(n)$ and $P$ values are presented on graphs. Ac, acute infection; BL, baseline (preinfection pre-HFD); Chr, chronic infection; Fat, preinfection post-HFD.

Local inflammatory infiltrates at multiple tissue sites contribute to the systemic immune activation and inflammation observed after HFD administration to SIV-infected NHPs and may explain the drastic $\mathrm{CD}^{+} \mathrm{T}$ cell loss, as well as the diminished chronic $\mathrm{CD} 4^{+} \mathrm{T}$ cell recovery observed in HFD-receiving AGMs. Mucosal $\mathrm{CD} 4^{+} \mathrm{T}$ cells normally recover in AGMs during chronic SIV infection (8). Consequently, our results suggest that diet may represent an overlooked contributor to the poor immune restoration in HIV-infected subjects on ART and should be integrated as a potential endpoint in trials aimed at controlling immune activation and inflammation and improving $\mathrm{CD}^{+} \mathrm{T}$ cell restoration.

Damage to the intestinal mucosa is also a major source of systemic immune activation and inflammation in chronic and longterm treated HIV/SIV infection. HFD induced significant changes in the intestinal immune environment and immune cell activation, promoting mucosal barrier destruction and increasing microbial translocation. Notably, Tregs were decreased in both HFD-receiving AGMs and PTMs, which may contribute to increased mucosal immune activation, inflammation, and autoimmunity (37).

Along with directly affecting the intestinal epithelium and shifting the mucosal environment toward a proinflammatory milieu (38), HFD may also have boosted the loss of total intestinal $\mathrm{CD}^{+} \mathrm{T}$ cells in HFD-receiving AGMs.

Based on previous literature (39-41), it is conceivable that the HFD also had an impact on the gut environment through changes in the microbiota. The very small sample sizes in our study prevented enough statistical power to draw meaningful conclusions in this area, however, and future studies of larger cohorts may provide more insight into the influence of the HFD on the mucosal microbiome and the consequences on the gut health and the natural history of SIV infection of such changes.

Regardless of the mechanism, it is clear that increases in the gut damage induced by HFD boost systemic immune activation and inflammation, thus promoting HIV/SIV disease progression in the HFD-treated AGMs and PTMs.

Metabolic and liver pathology are understudied aspects of HIV/SIV pathogenesis, yet they may have a critical clinical impact on drug metabolism and long-term response to ART (42). HFD clearly induced significant liver pathology in both AGMs and PTMs, illustrated by the high-grade liver steatosis, sinusoidal fibrosis, and elevations of plasma biomarkers for liver fibrosis. This information demonstrates that the HFD used here is relevant and has effects comparable to the HFD of human subjects, which similarly rapidly develop NAFLD. Liver lesions were much more frequent in the HFD-receiving SIV-infected PTMs compared with controls, indicating a synergistic negative impact of SIV infection and dietary habits on the liver.

Consequential to both HFD-induced gut and liver pathology, the observed changes in coagulation markers, along with elevated HDL oxidation, strongly confirm that HFD poses a serious threat to CV health and mortality in SIV/HIV infections. Myocardial, pericardial, and epicardial inflammation, myocardial cytolysis, microthrombi formation, and myocardial fibrosis occurred early and were severe in HFD-receiving PTMs compared with chronic SIVsab- 


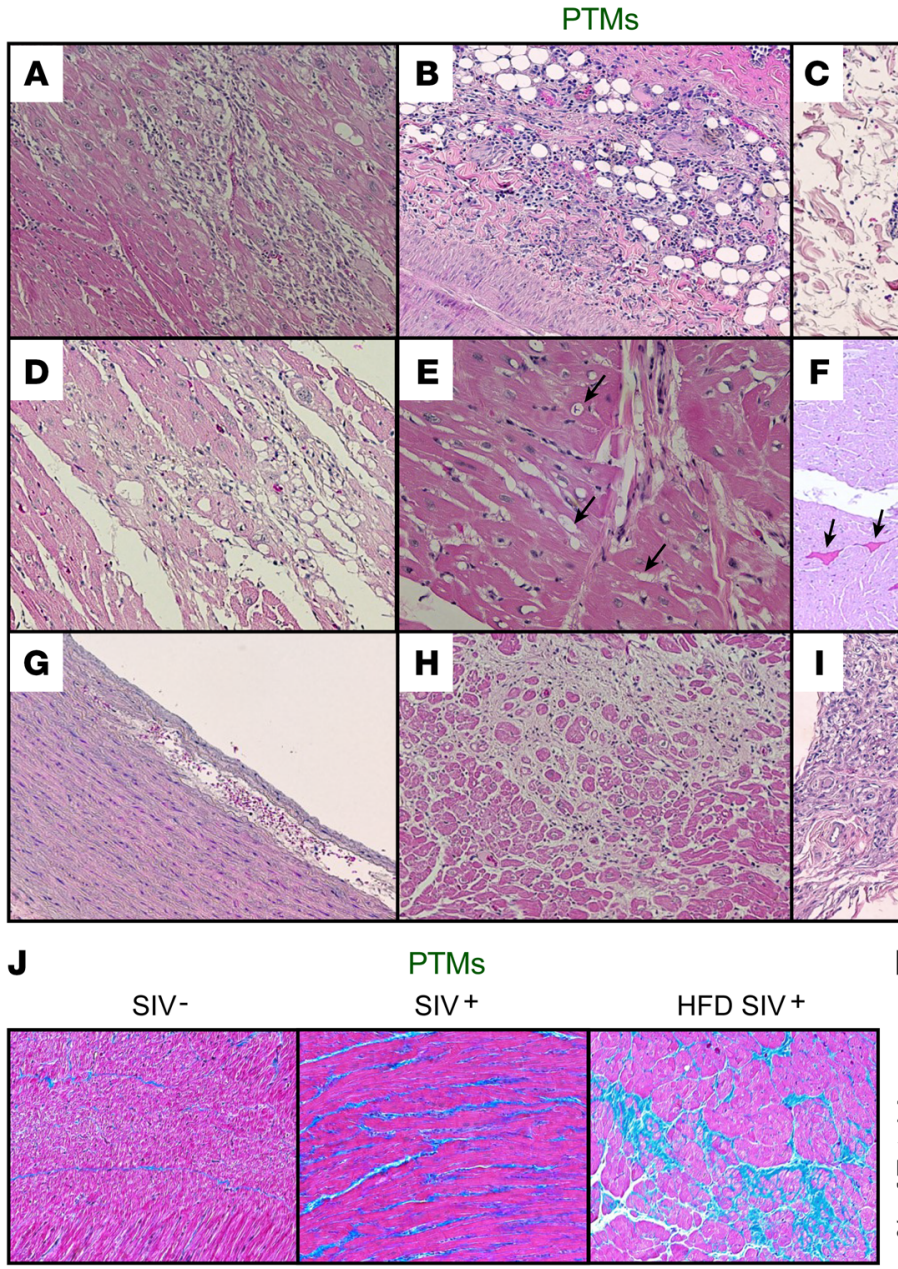

$\mathbf{L}$

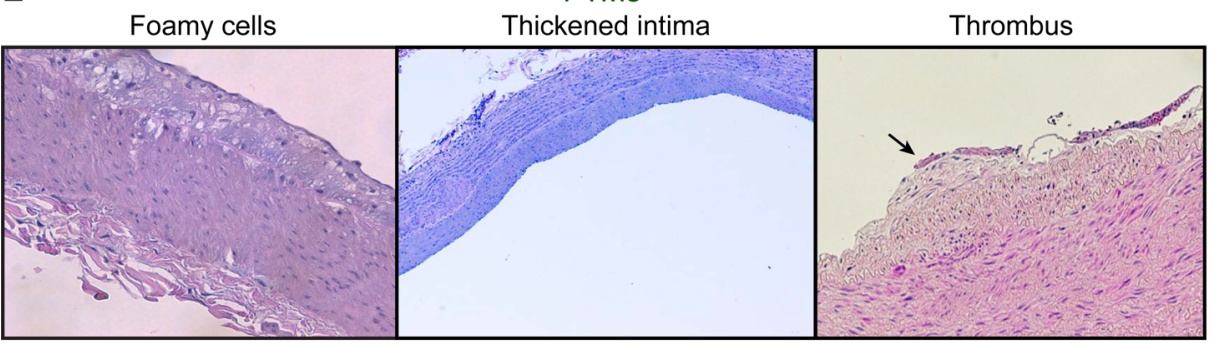

Figure 10. HFD increases severity of CV lesions in SIV-infected PTMs. Representative H\&E images of (A) myocardial and (B) epicardial immune infiltration, (C) lymphoid aggregates in epicardium, (D) myocardial cytolysis, (E) myocardial fatty infiltration (arrows), (F) microthrombi (arrows), (G) aortic dissection, (H) myocardial and (I) pericardial fibrosis in HFD-receiving PTMs. (J) Representative trichrome-stained images of cardiac fibrosis observed in HFD-receiving infected PTMs compared with control-infected and SIV- PTMs. Quantification of the percent area of collagen is shown in (K). Data were compared with Kruskal-Wallis test corrected for multiple comparisons and are presented as individual values with median. Sample size ( $n$ ) and $P$ values are presented on graphs. SIV'-, preinfection pre-HFD; SIV+, chronic infection without HFD; HFD SIV ${ }^{+}$, chronic infection with HFD. (L) Representative H\&E images of signs of evolving atherosclerosis with organized thrombus in HFD-receiving PTMs. Original magnifications: $\times 200$. infected controls. Most HFD-receiving PTMs also presented with incipient atherosclerosis (fatty streaks, foamy cells, and carotid intima thickening). These findings strongly imply an increased CV risk in HIV-infected subjects with unhealthy dietary habits.

Altogether, our results suggest that HFD may have a critical impact in disturbing the immunometabolic axes between gut, liver, and the CV system by inducing lesions and proinflammatory responses at all these sites.

Notably, the HFD effects on SIV pathogenesis were more clear cut in AGMs than in PTMs, likely due to the nature of SIV infection in the 2 models. The very severe features of untreated SIV infection in PTMs (massive and dramatic CD $4^{+} \mathrm{T}$ cell depletion, high and intrinsic microbial translocation [ref. 30], chronic immune activation and inflammation), likely masked the more subtle effects of diet. Nevertheless, the HFD deleterious effects on PTM survival were indisput- able and documented by a plethora of tissue lesions. Meanwhile, in nonpathogenic infections of AGMs, HFD clearly affected canonical biomarkers of HIV/SIV pathogenesis and tissue pathology, and even induced disease progression characterized by alterations in the many predictor biomarkers of AIDS, multiple opportunistic infections, and comorbidities. SIV disease progression is extremely rare in AGMs (35). In our 20 years of studying hundreds of SIV-infected AGMs, this is the first instance in which we observed SIV disease progression in an AGM as a result of an experimental intervention $(8,35,43)$. Our results indicate that the nonpathogenic models may be preferable to pathogenic models for studying the effects of diet on untreated SIV infection. Nevertheless, to model the majority of HIV-infected subjects, in which the virus is therapeutically suppressed, and to investigate the HFD-ARV interactions, SIV-infected PTMs on ART are the model of choice for our future studies. 
In conclusion, we demonstrated for the first time an important role of HFD in promoting the viral reservoir seeding and profound alterations of the gut mucosal integrity as well as hepatic and CV pathology. These features had a significant impact on SIV disease progression, development of comorbidities, and survival in both pathogenic and nonpathogenic SIV infections. We therefore propose a paradigm in which, through multiple pathological pathways, HFD accelerates SIV disease progression.

In the post-ART era, the major concerns in long-term virally suppressed subjects are no longer viral replication and disease progression. Instead, these issues comprise the non-AIDS comorbidities, residual immune activation and inflammation, and reservoir persistence. Our study strongly supports accounting for diet and other behavioral factors in future strategies aimed at reducing comorbidities, inducing functional cure and improving the quality of life for HIV-suppressed patients.

\section{Methods}

Animals, infection, and diet. Thirteen AGMs and 15 PTMs were infected with i.v. plasma equivalent to 300 tissue culture infectious doses (TCID50) of SIVsab $(25,43)$. For AGM infection, we used SIVsab92018 (43), a plasma stock containing a diverse swarm of transmitted-founder SIVsab strains, collected from an acutely infected AGM (44). PTM infection with SIVsab92018 ranges from rapid progression to virus control (25). Therefore, PTMs were infected with plasma from an SIVsab92018-infected rapid progressor PTM (SIVsabBH66) (25), then passaged in a naïve PTM, and collected at the viral peak. Thus, SIVsabBH66 contains transmitted-founder variants, while showing consistent high viral replication and pathogenicity in PTMs. The sequences of these 2 stocks are very similar, and the sole reason for using different stocks in the 2 species is the need for consistent viral replication.

The animal groups are described in the Results section, under the Study Design heading.

Commercially available primate HFD (Custom Diet 5A1F, Test Diet) and normal chow (ND) (Monkey Diet no. 5038, Lab Diet) were used for HFD and control groups, respectively. HFD and ND compositions are detailed in Table 1 . The HFD group received higher amounts of fat, much higher cholesterol content, a higher linoleic acid (omega-6 fatty acid) to linolenic acid (omega-3 fatty acid) ratio, and lower amounts of other omega-3 fatty acids, being therefore rich in several categories of fat dietary components reported as detrimental for human health.

Sample collection. Blood, LN, intestinal, and liver resections were collected and processed as described $(8,43)$. Collection and processing were similar for all the NHPs.

For HFD-receiving PTMs (Figure 1), blood and LNs were collected pre-HFD/pre-SIV infection, at 39 days post-HFD/pre-SIV infection, during the acute SIV infection (9 dpi), chronic infection ( $\approx 100 \mathrm{dpi})$, and at necropsies (128-218 dpi). Additional blood samples were collected at necropsy. Intestinal and liver resections were performed pre-HFD/preSIV infection and at 48 days post-HFD, during the acute SIV infection ( 9 dpi). Additional gut and liver samples were collected at necropsy.

The blood sampling schedule for both contemporary and historical PTM controls (Figure 1) was identical to the HFD-receiving PTMs, except for the post-HFD/pre-SIV infection time point. Gut and liver sampling were similar in the contemporary controls to HFD-receiving NHPs. Intestinal and liver resections were not performed in the historical controls.
At necropsy, numerous tissues were collected from both HFD-fed and chow-fed PTMs: gut, LNs, liver, lungs, kidneys, heart, aortas, coronaries, brachial, and carotid arteries.

In HFD-receiving AGMs (Figure 1), blood and LNs were collected pre-HFD/pre-SIV infection, at 102 days post-HFD/pre-SIV infection, during acute (9 dpi) SIV infection, and during chronic infection $(\approx 100$ dpi). Intestinal and liver resections were performed pre-HFD/pre-SIV infection, and at 82 days post-HFD/pre-SIV infection. Only 1 of the HFD-fed AGMs was euthanized during the follow-up, from which we collected the same tissues as from PTMs.

All AGM controls (Figure 1) were historical and followed an identical blood and LN sampling schedule as the HFD-receiving AGMs, except for the post-HFD/pre-SIV infection point. No gut and liver resections were performed in control AGMs.

Tissues collected at necropsy were fixed in 10\% buffered formalin, embedded in paraffin, and cut into $4-\mu \mathrm{m}$ sections for staining.

To reflect the differences in sampling between historical and contemporary controls, for each measurement, the exact number of animals is indicated in the figures or figure legends.

Flow cytometry analysis. Whole blood or mononuclear cells isolated from blood and intestinal samples were stained for flow cytometry, as described $(25,43)$. The mAbs are summarized in Supplemental Table 3. All Abs were validated and titrated using AGM and PTM PBMCs $(25,43)$. Stained cells were analyzed with an LSRII flow cytometer (BD Biosciences) and FlowJo Version 7.6 software (TreeStar). Gating strategies for immune cell populations and their activation status are described in the Supplemental Materials. Some flow cytometry stainings, such as Glut-1, CD80, CD86, IL-17, FoxP3, and CD25, were not done in the historical controls because they were not part of the original design of the studies including those animals. To avoid comparisons between stainings performed on fresh and frozen cells, we used only the flow cytometry results obtained on fresh samples (2-hour intervals after blood collection). Sample size is indicated for each measurement in the figures and figure legends.

Viral quantification. SIV pVLs were quantified using an SIVsab-specific quantitative real-time PCR (43). Cell-associated vRNA and vDNA were quantified by quantitative PCR (q-PCR). Total RNA was extracted from cell pellets using a TRIzol-based protocol (45). The DNA phase was extracted by adding $500 \mu \mathrm{L}$ of DNA extraction solution (4M Guanidine Thiocyanate, $50 \mathrm{mM}$ sodium citrate $\mathrm{NaCi}, 1-\mathrm{M}$ Tris) (45). Extracted plasma RNA samples were reverse-transcribed, and cDNAs and vDNAs were quantified by q-PCR using long terminal repeat-specific primers and a labeled probe (43). To assess the sample cell counts, CCR5-specific primers were used, and the final number of vRNA and vDNA copies was divided by the number of cells present in the sample to establish the number of vRNA copies/million cells.

CMV plasma VLs were quantified as follows: CMV genomic DNA was extracted from $400 \mu$ l of plasma using QIAmp DNA Mini kit (Qiagen). CMV DNA was quantified by q-PCR using the forward primer (5'-GTT TAG GGA ACC GCC ATT CTG-3' ${ }^{\prime}$, the reverse primer 5'-GTA TCC GCG TTC CAA TGC A-3', and the probe 5' 6FAM/TCC AGC CTC/ZEN/CAT AGC CGG GAA GG/3'IABkFQ, designed to recognize a region of $108 \mathrm{bp}$ in the immediate early gene of CMV (46).

Ten microliters of the plasma eluate was added to a PCR mix containing $300 \mathrm{nM}$ of forward and reverse primer, $100 \mathrm{nM}$ of probe, and 25 $\mu \mathrm{l}$ of TaqMan Gene Expression Master Mix (Thermo Fisher Scientific). The cycling parameters were $95^{\circ} \mathrm{C}$ for 5 minutes, followed by 45 cycles 
of amplification at $95^{\circ} \mathrm{C}$ for 15 seconds and 1 minute at $60^{\circ} \mathrm{C}$. CMV DNA quantification was performed using 10 -fold serial dilution of a plasmid containing the entire rhesus CMV immediate-early gene (46).

For the detection of CMV in tissues, the DNA was extracted from the snap-frozen tissues by TRIzol isolation (Sigma-Aldrich), and 700 ng of total nucleic acid was then subjected to PCR using the primers and conditions employed in the real-time PCR assay.

Histological analysis. Gut, LNs, liver, lungs, brain, kidneys, heart, aortas, coronaries, and carotids collected at the necropsy were first stained with H\&E for routine histopathology (3). Infection with Cystoisospora belli was confirmed by Giemsa stain (47). The degree of liver and heart fibrosis was assessed by Masson trichrome (3). Immunohistochemistry was performed as described in the Supplemental Material $(8,45)$ to assess neutrophil infiltration and for CMV detection. Slides were visualized with a Carl Zeiss Axio Imager M1 microscope using the following objectives: $\times 10$ /0.3 DICI Plan Neofluar, $\times 20$ /0.8 DICII Plan Apochromat, and $\times 40$ /0.75 DICII Plan Neofluar. Micrographs were taken with a Carl Zeiss AxioCam MRc5. Images were acquired and analyzed using Carl Zeiss Axio Vision SE64 Release 4.8.2. Quantification was performed using open-source FIJI image software using 10 images per section/time point/animal. The positive signal was isolated via color threshold, and the percent area positive was measured and averaged.

Measurement of plasma/serum-based biomarkers. Microbial translocation was monitored by testing the plasma LPS levels with Limulus Amebocyte Lysate assay (Lonza), according to the manufacturer's protocol. Intestinal FABP (I-FABP) (48), was measured with a monkey I-FABP ELISA kit (MyBioSource, Inc.) (48). Plasma hyaluronic acid levels (32) were measured with Monkey Hyaluronic Acid ELISA kit (MyBioSource).

Inflammatory cytokines and chemokines were monitored in plasma (45), using the Cytokine Monkey Magnetic 29-Plex Panel (Invitrogen), as per manufacturer's instruction. Results were read by a Bio-Plex reader (Bio-Rad Laboratories), using Luminex technology (Luminex Corporation). CRP was tested using a monkey CRP ELISA Kit (Life Diagnostics) (45).

Coagulation status was assessed using multiple biomarkers associated with hypercoagulation in humans (49). TF levels were measured with IMUBIND Tissue Factor ELISA (Sekisui Diagnostics, LLC). D-dimer was measured using a STAR automated coagulation analyzer, (Diagnostica Stago) and an immunoturbidimetric assay (Liatest D-DI; Diagnostica Stago) (3). Prompted by our previous study showing that TF pathway may be critical in bridging hypercoagulation and inflammation in HIV/SIV pathogenesis (20), we measured the plasma levels of factor VII using a commercially available ELISA assay (Affinity Biologicals).

To evaluate the CV risk, serum lipid composition was also assessed by measuring cholesterol (Marshfield Labs), oxidized HDL in plasma with OxiSelect Human Oxidized HDL ELISA kit (Cell Biolabs, Inc.). Troponin-I, a biomarker of cardiac stress, which is elevated in chronically HIV-infected subjects (50), was measured with ultrasensitive monkey cardiac troponin-I ELISA (Life Diagnostics). Platelet and endothelial activation were assessed by measuring the plasma levels of soluble p-selectin (Platinum ELISA, eBioscience, Inc.) and soluble ICAM-1 (ICAM-1 Monkey Instant ELISA Kit, Thermo Fisher Scientific).
Statistics. All statistical analyses were performed using Prism 5.0 software (GraphPad Software) and R (R Foundation for Statistical Computing, Vienna, Austria). Data are represented as individual values with medians, unless otherwise specified. Due to the relatively small size of the groups and individual variations between NHPs, for some parameters we used baseline levels (average of 3 different time points collected before both HFD and SIV infection) to normalize the results as fold change from the baseline. Different time points for the HFD group (pre-, post- HFD, acute and chronic SIV) were matched within animals, and comparisons were made using the Friedman test (for 1-way repeated measurements by rank) with post hoc comparisons with baseline corrected for multiple comparisons $(51,52)$. Comparisons for the same time point between the independent samples of the HFD and control groups were performed with the Kruskal-Wallis test. Survival was estimated by the Kaplan-Meier estimator, and compared between groups with the Mantel-Cox method. $P$ values less than 0.05 were considered significant.

Study approval. This study was reviewed and approved by the Institutional Animal Care and Use Committee (protocols no. 12121250 and no. 14043645) of the University of Pittsburgh at Pittsburgh, Pennsylvania, USA.

\section{Author contributions}

IP and CA designed and oversaw the study. TH, BBP, and KDR contributed to sample processing. GHR and TD provided veterinarian care, administered diets, and collected samples. TH and CX performed flow cytometry experiments and analyses. TH, NK, EF, WMJ, and KM performed histologies, IHC stainings, and quantifications. SD and DF performed exploratory microbiome measurements and analyses. TH performed the soluble biomarker measurements. PS, $\mathrm{EP}$, and BBP performed viral quantifications. RPT provided coagulation factor measurements. RMR performed statistical analyses. TH generated figures and participated in the data analyses. IP performed histological diagnoses. RPT, CW, and AL helped with study design, data interpretation, and discussion. IP, CA, TH, DF, RPT, CW, AL, and RMR wrote and edited the manuscript.

\section{Acknowledgments}

This study was funded through NIH/NHLBI/NIAID/NIDDK/ NCRR R01 grants HL117715 (to IP), HL123096 (to IP), AI119346 (to CA), DK113919 (to IP and CA), DK119936 (to CA), RR025781 (to CA and IP), and AI104373 (to RMR). RMR was funded by grant PTDC/ MAT-APL/31602/2017 from the Fundacao para a Ciencia e Tecnologia (Portugal). DNF and CCW were supported by the University of Colorado GI and Liver Innate Immunity Program. KDR and BBP were partly supported by the NIH Training Grant T32AI065380. The funders had no role in study design, data collection and analysis, decision to publish, or preparation of the manuscript.

Address correspondence to: Ivona Pandrea, S739 Scaife Hall, Department of Pathology, 3550 Terrace Street, Pittsburgh, Pennsylvania 15261, USA. Phone: 412.383.5834; Email:pandrea@pitt.edu.
1. Pandrea I, Apetrei C. Where the wild things are: pathogenesis of SIV infection in African nonhuman primate hosts. Curr HIV/AIDS Rep. 2010;7(1):28-36.
2. Kuller LH, et al. Inflammatory and coagulation biomarkers and mortality in patients with HIV infection. PLoS Med. 2008;5(10):e203.

3. Pandrea I, et al. Coagulation biomarkers predict disease progression in SIV-infected nonhuman primates. Blood. 2012;120(7):1357-1366.

4. Pandrea I, Landay A, Wilson C, Stock J, Tracy R, Apetrei C. Using the pathogenic and nonpatho- 
genic nonhuman primate model for studying non-AIDS comorbidities. Curr HIV/AIDS Rep. 2015;12(1):54-67.

5. He T, Falwell E, Brocca-Cofano E, Pandrea I. Modeling aging in HIV infection in nonhuman primates to address an emerging challenge of the post-ART era. Curr Opin Virol. 2017;25:66-75.

6. Brenchley JM, et al. Microbial translocation is a cause of systemic immune activation in chronic HIV infection. Nat Med. 2006;12(12):1365-1371.

7. Lurain NS, Hanson BA, Hotton AL, Weber KM, Cohen MH, Landay AL. The association of human cytomegalovirus with biomarkers of inflammation and immune activation in HIV-1-infected women. AIDS Res Hum Retroviruses. 2016;32(2):134-143.

8. Pandrea IV, et al. Acute loss of intestinal $\mathrm{CD} 4^{+} \mathrm{T}$ cells is not predictive of simian immunodeficiency virus virulence. JImmunol. 2007;179(5):3035-3046.

9. Trichopoulou A, Costacou T, Bamia C, Trichopoulos D. Adherence to a Mediterranean diet and survival in a Greek population. $N$ Engl J Med. 2003;348(26):2599-2608.

10. Kahn SE, Hull RL, Utzschneider KM. Mechanisms linking obesity to insulin resistance and type 2 diabetes. Nature. 2006;444(7121):840-846.

11. Kim KA, Gu W, Lee IA, Joh EH, Kim DH. High fat diet-induced gut microbiota exacerbates inflammation and obesity in mice via the TLR4 signaling pathway. PLoS One. 2012;7(10):e47713.

12. Müller VM, et al. Gut barrier impairment by highfat diet in mice depends on housing conditions. Mol Nutr Food Res. 2016;60(4):897-908.

13. Frank DN, et al. Perilipin-2 modulates lipid absorption and microbiome responses in the mouse intestine. PLoS One. 2015;10(7):e0131944.

14. Shi L, et al. The prevalence of nonalcoholic fatty liver disease and its association with lifestyle/ dietary habits among university faculty and staff in Chengdu. Biomed Environ Sci. 2012;25(4):383-391.

15. Sobrecases H, et al. Effects of short-term overfeeding with fructose, fat and fructose plus fat on plasma and hepatic lipids in healthy men. Diabetes Metab. 2010;36(3):244-246.

16. Wu J, Zhang $\mathrm{H}$, Zheng $\mathrm{H}$, Jiang Y. Hepatic inflammation scores correlate with common carotid intima-media thickness in rats with NAFLD induced by a high-fat diet. BMC Vet Res. 2014;10:162.

17. Li C, et al. High-fat diet amplifies renal renin angiotensin system expression, blood pressure elevation, and renal dysfunction caused by Ceacam1 null deletion. Am J Physiol Endocrinol Metab. 2015;309(9):E802-E810.

18. Suzuki M, et al. High fat and high fructose diet induced intracranial atherosclerosis and enhanced vasoconstrictor responses in non-human primate. Life Sci. 2006;80(3):200-204.

19. Cleuren AC, et al. Changes in dietary fat content rapidly alters the mouse plasma coagulation profile without affecting relative transcript levels of coagulation factors. PLoS One. 2015;10(7):e0131859.

20. Schechter ME, et al. Inflammatory monocytes expressing tissue factor drive SIV and HIV coagu- lopathy. Sci Transl Med. 2017;9(405):eaam5441.

21. Wang T, Yi R, Green LA, Chelvanambi S, Seimetz M, Clauss M. Increased cardiovascular disease risk in the HIV-positive population on ART: potential role of HIV-Nef and Tat. Cardiovasc Pathol. 2015;24(5):279-282.

22. Mansfield KG, et al. A diet high in saturated fat and cholesterol accelerates simian immunodeficiency virus disease progression. J Infect Dis. 2007;196(8):1202-1210.

23. Zhang $\mathrm{R}$, et al. The difference in nutrient intakes between Chinese and Mediterranean, Japanese and American diets. Nutrients. 2015;7(6):4661-4688.

24. Beaumier CM, et al. CD4 downregulation by memory $\mathrm{CD}^{+} \mathrm{T}$ cells in vivo renders African green monkeys resistant to progressive SIVagm infection. Nat Med. 2009;15(8):879-885.

25. Mandell DT, et al. Pathogenic features associated with increased virulence upon Simian immunodeficiency virus cross-species transmission from natural hosts. J Virol. 2014;88(12):6778-6792.

26. Wang ZD, et al. Prevalence of Cryptosporidium, microsporidia and Isospora infection in HIVinfected people: a global systematic review and meta-analysis. Parasit Vectors. 2018;11(1):28.

27. $\mathrm{Wu} \mathrm{H}$, et al. $\mathrm{T}$-cell accumulation and regulated on activation, normal $\mathrm{T}$ cell expressed and secreted upregulation in adipose tissue in obesity. Circulation. 2007;115(8):1029-1038.

28. Damouche A, et al. Adipose tissue is a neglected viral reservoir and an inflammatory site during chronic HIV and SIV infection. PLoS Pathog. 2015;11(9):e1005153.

29. Kleinman AJ, Sivanandham R, Pandrea I, Chougnet CA, Apetrei C. Regulatory T cells as potential targets for HIV cure research. Front Immunol. 2018;9:734.

30. Klatt NR, et al. Compromised gastrointestinal integrity in pigtail macaques is associated with increased microbial translocation, immune activation, and IL-17 production in the absence of SIV infection. Mucosal Immunol.2010;3(4):387-398.

31. Wong VW, et al. Bacterial endotoxin and nonalcoholic fatty liver disease in the general population: a prospective cohort study. Aliment Pharmacol Ther. 2015;42(6):731-740.

32. Halfon $\mathrm{P}$, et al. Accuracy of hyaluronic acid level for predicting liver fibrosis stages in patients with hepatitis C virus. Comp Hepatol. 2005;4:6.

33. Sacks FM, et al. Dietary fats and cardiovascular disease: a presidential advisory from the American Heart Association. Circulation. 2017;136(3):e1-e23.

34. Ru D, et al. Oxidized high-density lipoprotein accelerates atherosclerosis progression by inducing the imbalance between Treg and teff in LDLR knockout mice. APMIS. 2015;123(5):410-421.

35. Pandrea I, Silvestri G, Apetrei C. AIDS in African nonhuman primate hosts of SIVs: a new paradigm of SIV infection. Curr HIV Res. 2009;7(1):57-72.

36. Whitney JB, et al. Rapid seeding of the viral reservoir prior to SIV viraemia in rhesus monkeys. Nature. 2014;512(7512):74-77.

37. Luu M, Steinhoff U, Visekruna A. Functional heterogeneity of gut-resident regulatory T cells. Clin Transl Immunology. 2017;6(9):e156.
38. Schirmer M, et al. Linking the human gut microbiome to inflammatory cytokine production capacity. Cell. 2016;167(4):1125-1136.e8.

39. Cani PD, et al. Changes in gut microbiota control metabolic endotoxemia-induced inflammation in high-fat diet-induced obesity and diabetes in mice. Diabetes. 2008;57(6):1470-1481.

40. David LA, et al. Diet rapidly and reproducibly alters the human gut microbiome. Nature. 2014;505(7484):559-563.

41. Pedersen R, Andersen AD, Hermann-Bank ML, Stagsted J, Boye M. The effect of high-fat diet on the composition of the gut microbiota in cloned and non-cloned pigs of lean and obese phenotype. Gut Microbes. 2013;4(5):371-381.

42. Ahmed MH, Husain NE, Malik A, Woodward C, Mital D. Non-alcoholic fatty liver disease and HIV/AIDS: a new way of modulation of cardiovascular risk. AIDS Rev. 2017;19(4):190-197.

43. Pandrea I, et al. Simian immunodeficiency virus SIVagm.sab infection of Caribbean African green monkeys: a new model for the study of SIV pathogenesis in natural hosts. J Virol. 2006;80(10):4858-4867.

44. Gnanadurai CW, et al. Genetic identity and biological phenotype of a transmitted/founder virus representative of nonpathogenic simian immunodeficiency virus infection in African green monkeys. JVirol. 2010;84(23):12245-12254.

45. Pandrea I, et al. Antibiotic and antiinflammatory therapy transiently reduces inflammation and hypercoagulation in acutely SIV-infected pigtailed macaques. PLoS Pathog. 2016;12(1):e1005384.

46. Kaur A, Hale CL, Noren B, Kassis N, Simon MA, Johnson RP. Decreased frequency of cytomegalovirus (CMV)-specific CD4 $4^{+} \mathrm{T}$ lymphocytes in simian immunodeficiency virus-infected rhesus macaques: inverse relationship with CMV viremia. J Virol. 2002;76(8):3646-3658.

47. Yen JH, Huang HS, Chuang CJ, Huang ST. Activation of dynamin-related protein 1 - dependent mitochondria fragmentation and suppression of osteosarcoma by cryptotanshinone. J Exp Clin Cancer Res. 2019;38(1):42.

48. He T, et al. Critical role for the adenosine pathway in controlling Simian immunodeficiency virus-related immune activation and inflammation in gut mucosal tissues. J Virol. 2015;89(18):9616-9630.

49. Mayne E, et al. Increased platelet and microparticle activation in HIV infection: upregulation of Pselectin and tissue factor expression. J Acquir Immune Defic Syndr. 2012;59(4):340-346.

50. Bello A, et al. Troponin I as marker of subclinica cardiac injury in human immunodeficiency virus-seropositive patients in Sokoto, Nigeria. J HIV Hum Reprod. 2016;4(1):20-26.

51. Hollander M, Wolfe DA. Nonparametric Statistical Methods, Solutions Manual. 2nd ed. Hoboken, New Jersey, USA; Wiley: 1999.

52. Pohlert T. PMCMRplus: Calculate Pairwise Multiple Comparisons of Mean Rank Sums Extended. R package version 1.4.1. 2018. R-Project. https://cran.r-project.org/web/packages/PMCMRplus/PMCMRplus.pdf. Published November 12, 2018. Accessed October 10, 2019. 